\title{
Chop/Ddit3 depletion in $\beta$-cells alleviates ER stress and corrects hepatic steatosis
}

Jing Yong1, Vishal S. Parekh², Jonamani Nayak', Zhouji Chen¹, Cynthia Lebeaupin $^{1,3}$, Jiangwei Zhang ${ }^{4}$, Thazha P. Prakash ${ }^{4}$, Sue Murray ${ }^{4}$, Shuling Guo ${ }^{4}$, Julio E. Ayala ${ }^{5,6}$, Leslie S. Satin², Randal J. Kaufman ${ }^{1, *}$

${ }^{1}$ Degenerative Diseases Program, Sanford-Burnham-Prebys Medical Discovery Institute, 10901 N. Torrey Pines Rd., La Jolla, CA 92037

2 Department of Pharmacology, University of Michigan Medical School, 1000 Wall St., Ann Arbor, MI 48105

3 Department of Medicine, University of California San Diego, La Jolla, CA 92093

4 Department of Antisense Drug Discovery, Ionis Pharmaceuticals, Inc., 2855 Gazelle Court, Carlsbad, CA 92010

5 Cardiometabolic Phenotyping Core, Sanford-Burnham Medical Research Institute, 6400 Sanger Road, Orlando, FL 32827

${ }^{6}$ Department of Molecular Physiology \& Biophysics, Vanderbilt University School of Medicine, Nashville, Tennessee, USA

* Corresponding author: rkaufman@sbpdiscovery.org 


\section{Abstract}

Type 2 diabetes (T2D) is a metabolic disorder characterized by hyperglycemia, hyperinsulinemia and insulin resistance (IR). During the early phase of T2D, insulin synthesis and secretion by pancreatic $\beta$ cells is enhanced, which can lead to proinsulin (Prolns) misfolding that aggravates endoplasmic reticulum (ER) homeostasis in $\beta$ cells. Moreover, increased insulin in the circulation may contribute to fatty liver disease. Medical interventions aimed at alleviating ER stress in $\beta$ cells while maintaining optimal insulin secretion are therefore an attractive therapeutic strategy for T2D. Previously, we demonstrated that germline Chop gene deletion preserved $\beta$ cells in high fat diet (HFD) fed mice and in leptin receptor-deficient $d b / d b$ mice. In the current study, we further investigated whether targeting Chop/Ddit3 specifically in murine $\beta$ cells confers therapeutic benefits. First, we show that Chop deletion in $\beta$ cells alleviates $\beta$ cell ER stress and delays glucose-stimulated insulin secretion (GSIS) in HFD fed mice. Second, importantly, $\beta$ cell-specific Chop deletion prevented liver steatosis and hepatomegaly in aged HFD fed mice without affecting basal glucose homeostasis. Third, we provide the first mechanistic evidence that ER remodeling secondary to Chop deletion modulates glucose-induced islet $\mathrm{Ca}^{2+}$ oscillations. Finally, using state-of-the-art GLP1-conjugated Chop AntiSense Oligonucleotides (GLP1-Chop ASO), we demonstrated that the Chop deletion induced GSIS change is a long term complex event in $\beta$ cells. In summary, our results demonstrate that Chop depletion in $\beta$ cells is a new therapeutic strategy to alleviate dysregulated insulin secretion and the consequently fatty liver disease in T2D.

(245 words) 
1 Type 2 diabetes (T2D) is a metabolic disorder that poses a severe health

2 challenge for modern society as it is estimated by the United States' Centers for

3 Disease Control and Prevention that thirty million Americans are affected by this

4 condition (1). T2D is characterized by insulin resistance (IR), hyperglycemia and

5 hyperinsulinemia (2). At the same time, current T2D therapeutics focus on

6 achieving improved blood glucose homeostasis by improving both insulin

7 secretion and reducing peripheral IR. Pharmacological interventions such as

8 glitazones and glucagon-like peptide (GLP1) receptor agonists are limited in that

9 despite achieving glucose control, there is insufficient clinical evidence to support

10 a beneficial effect on human pancreatic $\beta$ cells (3).

11 Pancreatic $\beta$ cell pathogenesis coupled with peripheral IR has been the 12 traditional explanation for T2D, although recent studies using mouse models and 13 clinical findings made in the Pima Indians (4) support an alternative hypothesis 14 that hyperinsulinemia can serve as a driving force for IR in mouse T2D models (5, 15 6). In the Mehran and Johnson paradigm (5), excessive amounts of insulin 16 secreted by pancreatic $\beta$ cells is the cause of peripheral IR and fatty liver 17 development (7). In this model, reducing the insulin load would, therefore, 18 alleviate IR. However, experimental observations made using murine Insulin gene $\mathrm{KO}$ models may not be relevant to human $\mathrm{T} 2 \mathrm{D}$, although a moderate

20 reduction in insulin production may be beneficial (7). Nevertheless, it is 21 technically challenging to accurately manipulate insulin mRNA levels (8), 22 especially since it is the most abundant $\beta$ cell mRNA, accounting for $\sim 30 \%$ of the 23 total transcriptome in mature $\beta$ cells based on our own RNA-Seq data (9) and 24 other's findings $(10,11)$. Therefore, no current means exist to directly evaluate 25 whether a reduction in insulin expression can achieve beneficial metabolic effects.

26 On the other hand, previous studies demonstrated that germline deletion of Chop 27 (also known as Ddit3/Gadd153) prevents $\beta$ cell failure in diabetes models (12-14). 28 It is unknown, however, whether Chop deletion protects in $\beta$ cells in a cell 29 autonomous manner, especially considering the role of $\mathrm{CHOP}$ in reducing weight 
30 gain (12), regulating hepatic lipid metabolism and suppressing adipocyte 31 development (13). To critically evaluate the metabolic consequence of deleting 32 Chop in $\beta$ cells, we therefore generated a conditional Chop deletion model by 33 breeding a floxed Ddit3 gene allele (15) with a RIP-CreERT transgene (16). In 34 this model, $\beta$ cell-specific Chop deletion is temporally controlled by tamoxifen 35 (TAM) injections (referred to hereafter as "Chop $\Delta / \Delta: R I P$-Cre" or simply "Chop $36 \beta K O "$ mice, see Sppl. Table I for breeding scheme).

37 To evaluate the primary effect of Chop deletion, we first assessed blood glucose and insulin levels in normal diet-fed male Chop BKO mice (with TAM administered to $21 \mathrm{wk}$-old mice), compared to the isogenic male littermates that received diluent as controls ( $\mathrm{n}=4 \mathrm{males} / \mathrm{group}$ ). Four months after Chop deletion,

41 Chop $\beta K O$ mice had similar fasting blood glucose levels and displayed similar 42 responses to a glucose challenge (i.p. at $1.5 \mathrm{mg}$ glucose/g weight, Fig. 1A) 43 compared to control littermates. Interestingly, fasting insulin levels were reduced, 44 although not significantly, while glucose stimulated insulin secretion (GSIS) was 45 dramatically blunted at 30min (Fig. 1B, p< 0.05 for 30min). In addition, we found 46 a trend of reduced $\beta$-cell function, by applying the Homeostasis Model Assessment of $\beta$-cell function (HOMA- $\beta$ ) (Fig. S1A, $p=0.06$ ), but not of HOMAIR (Fig. S1B, $\mathrm{p}=0.31$ ) (17) suggesting unchanged $\mathrm{IR}$ in Chop $\beta K O$ mice. Nonetheless, there was a significant linear correlation between fasting insulin and body weight (Fig. 1C, $\mathrm{p}<0.01$ by F-test). Furthermore, there was a trend towards decreased cumulative at 4 months (4mon) weight gain in Chop $\beta K O$

52 mice (Fig. 1D, $p=0.11$ ), which became significant when we included the nonCreERT littermates in comparison (Fig. S1C, $\mathrm{p}<0.05$ ). Importantly, cumulative weight gain as a function of time was not affected by CreERT gene expression as we previously reported (9) (and data not shown).

Given that male Chop $\beta K O$ mice had relatively normal phenotypes, we next

57 challenged them with a high-fat diet (HFD, 45\% fat in kcal) for 20wks starting

58 from an age of $\sim 9$ wks, with Chop deletion induced by TAM at 10wks post HFD

59 (scheme shown in Fig. 1E). In addition, we selected littermates harboring 
60 wildtype (WT) Chop alleles as the control group as they can be TAM treated.

61 Before Chop deletion, the two groups were metabolically indistinguishable with

62 no significant difference in body weight or blood glucose levels, either before or

63 10wks after the HFD (Fig. 1 F\&G). After Chop deletion, a moderate but 64 significant decrease in body weight was observed in Chop $\beta K O$ mice (Fig. 1F, $\mathrm{p}<$

650.01 at Wk20), which had no effect on blood glucose (Fig. 1G). After 20wks HFD 66 feeding, murine livers were dissected for visual inspection and for liver 67 triglyceride (TG) analysis. HFD feeding caused hepatomegaly and liver discoloration associated with fatty deposits in control littermates (Fig. 1H, black line), as well as for a Chop $\beta$ Het mouse in the litter (Fig. 1H, green line). In contrast, the three Chop $\beta K O$ mice had normal-sized livers and appeared healthy (Fig. 1H, red line). The morphological impression was further confirmed quantitatively by showing significantly reduced liver weight and TG content in Chop $\beta K O$ mice compared to littermates (Fig. $1 \mathbf{I}$ and $\mathbf{J}, \mathbf{p}<0.05$ for both). We surmised that chronically reduced $\beta$ cell insulin secretion may prevent fatty liver development in the HFD-fed C57BL/6 mice, as previously proposed by others (5, 6). Supporting this hypothesis, pancreatic insulin content (standardized by tissue wet weight) was reduced 3 -fold in the Chop $\beta K O$ mice (Fig. 1K, $\mathrm{p}=0.11$ due to variability in the control group) and was positively correlated with TG content (Fig. $1 \mathrm{~L}, \mathrm{p}<0.05$ by F-test). Echoing the recently reported human study ( 7 ), it will be of interest to investigate if the improvement of fatty liver we observed here reflects reduced de novo hepatic lipogenesis.

Intrigued by the blunted GSIS response and the protection from HFD-induced hepatic steatosis observed in the Chop $\beta K O$ mice (compare Fig. 1B vs. Fig. 1H), we further evaluated the mice to test whether the reduced pancreatic insulin content negatively affected glucose metabolism. For this purpose, a follow-up 22 wk HFD experiment was performed using male mice, during which body weight, food intake and non-fasting blood glucose levels were all monitored. We found no significant differences in these variables at any time point when comparing knockouts to their Chop $\beta$ Het littermates (Fig. 2 A to $\mathbf{C}$, respectively), while glucose tolerance in all the HFD fed mice exhibited a significantly increased 
91 glucose excursion from baseline, as expected ( $A U C$-IPGTT, Fig. 2D, $\mathrm{p}=0.0001$

92 for "HFD" by 2-way ANOVA). In the same experiment, Chop BKO HFD mice were more sensitive to insulin only after Chop deletion, although the difference was not statistically significant (compare Sppl. Fig. 2A and 2B, with TAM administered at 8wks post HFD). Similar to the insulin measurements made in the sera from mice fed normal diet (Fig. 1B), Chop $\beta K O$ HFD fed mice had slightly decreased serum C-peptide, both before and after glucose stimulation (Sppl. Fig. S2C).

As we observed no adverse effects of Chop deletion on mouse whole body metabolism, we performed a hyperglycemic clamp test on a different HFD cohort using mice of both sexes (Fig. 2E), again using age-matched Chop $\beta$ Het littermates (Chop +/-: RIP-Cre) as controls. While no differences were observed in body weight nor fasting blood glucose (Sppl. Table II), it was intriguing to observe that both insulin and C-peptide secretion in Chop $\beta K O$ mice appeared to be significantly slowed following hyperglycemia clamping, featuring a delayed, increased secretion after 40min (Fig. 2 F and $\mathbf{G}, \mathrm{p}<=0.01$ by RM 2-way ANOVA labeled). Notably, the control Chop $\beta$ Het mice showed basal hyperinsulinemia and reduced first phase responses to glucose demonstrating that the HFD model properly replicated the phenotype of humans during pre-diabetic phase and early T2D phase. Chop $\beta K O$ mice required a significantly higher glucose infusion rate (GIR) to maintain their blood glucose target at $~ 300 \mathrm{mg} / \mathrm{dL}(=16.7 \mathrm{mM})$ (Fig. 2 H and I), suggesting increased glucose clearance was due to increased insulin secretion and not altered insulin sensitivity ( $p=0.82$, Fig. 2J). Similarly, no difference was found in HOMA- $\beta$ nor HOMA-IR (Fig. S3 A and B, $p=0.75$ and 0.72 , respectively). Furthermore, the differences found in the hyperglycemic clamp study were not due to altered $\beta$ cell mass of the Chop $\beta K O$ mice, as no

$117\left(\right.$ Insulin $\left.^{+}\right)$areas (Fig. 2K, representative islet morphologies shown in Sppl Fig.

118 S4, with total surveyed pancreas areas reported in Sppl Fig. S5A). Similarly, no 119 differences were observed in the two groups with regards to $\alpha$ cell distribution in 120 the islets (Sppl. Fig. S4B vs. S4D), $\alpha$ cell mass, or the relative ratio of a cells to $121 \beta$ cells (Sppl. Fig. S5B-D). Finally, we ruled out the possibility of an indirect 
122 effect of $\beta$-cell Chop deletion on hepatic gluconeogenesis using a pyruvate

123 tolerance test (Sppl. Fig. S6). In contrast, an in vitro GSIS assay using germline 124 Chop KO islets confirmed a delayed, increased GSIS phenotype (Sppl. Fig. S7, $125 \mathrm{p}<0.01$ for "240 min"), pinpointing an islet-autonomous change in GSIS 126 accounting for our observations in whole animals.

127 To confirm that Chop deletion in $\beta$-cells reduces ER stress (12), we performed 128 molecular analysis of UPR markers using qRT-PCR and subsequently using 129 whole transcriptomic profiling by $\operatorname{mRNA}$-Seq $(9,18)$ on the RNA extracted from 130 the islets of male HFD fed mice. Molecular assays revealed greatly decreased 131 Insulin transcripts (Fig. 3A, 75\% reduction in Chop $\beta K O$ islets) associated with 132 reduced UPR markers, for example, Atf4, Bip and spliced Xbp1 (sXbp1) in $\beta K O$ 133 islets compared to WT littermates (Fig. 3B). Furthermore, supporting our findings 134 by qRT-PCR, transcriptome profiling showed significant reduction in other UPR 135 markers, represented by Atf3, Bip/Hspa5, Sec23b and ERdj4/Dnajb9 on a 136 "Volcano plot" (Fig. 3C). Interestingly, Chop deletion did not affect $\beta$ cell identity, 137 as the key $\beta$ cell transcription factors, including Pdx1, Nkx6.1, Mafa, Is/1 and 138 Ngn3, were not altered in Chop-deleted islets (Fig. 3D), consistent with our 139 histological findings of unchanged $\beta$-cell mass and islet morphology. In addition, 140 Chop deletion did not alter the mRNAs encoding Cpe, Glut2 or PC1/2 (Sppl. Fig. 141 S8), further supporting the hypothesis that Chop deletion did not impair $\beta$ cell 142 differentiation. In contrast, Wfs1, Gadd34/Ppp1r15a, and Bip/Hspa5 (encoded by 143 two alternatively spliced isoforms, namely NM_022310 and NM_001163434), 144 representing the UPR target genes for the IRE1 $\alpha$, PERK and ATF6a branches of 145 the UPR, respectively, were all found to be significantly reduced (Fig. 3E). 146 Reductions in UPR transcript abundance was supported further by our finding 147 that a subset of mRNAs that encode important ER proteins were altered (see 148 heat map in Fig. 3F), suggesting that CHOP has a long-term effect on "ER 149 remodeling". Since the UPR is an adaptive response to ER stress, we tested 150 biochemically if Chop deleted $\beta$ cells exhibit reduced ER stress, by probing the 151 interaction between Prolns and BiP, as BiP binding to misfolded proinsulin is the 152 gold-standard biochemical indicator for ER due to Prolns misfolding in the $\beta$ cell 
153 (19-21). By the BiP co-IP assay, we confirmed that less BiP protein was 154 associated with Prolns in the Chop $\beta K O$ islets (Fig. 3G, and data not shown), confirming reduced ER stress in Chop $\beta K O$ islets.

156 These results led us to hypothesize that under physiological conditions, CHOP 157 serves as a transcriptional hub in $\beta$ cells that can alter ER function by reducing 158 the expression of genes encoding ER-structural, -functional and ER-to-Golgi 159 trafficking proteins in order to accommodate Prolns synthesis (Fig. $\mathbf{3 H}$, Model). 160 Conversely, Chop deletion in $\beta$ cells decreased Prolns synthesis accompanied 161 by more efficient folding as indicated by less BiP interaction.

162 A missing mechanistic link, however, is that CHOP does not directly bind to the 163 promoter regions of all of these genes affected (Fig. 3F), as we demonstrated 164 previously using ChIP-Seq (18). We thus wondered how deleting Chop could 165 result in changes in ER function without altering the promoter activity of ER166 related genes. The $\mathrm{ER}$ is the major intracellular $\mathrm{Ca}^{2+}$ storage organelle, and both 167 ER and cytosolic $\mathrm{Ca}^{2+}$ and ATP-to-ADP levels can be profoundly affected by 168 physiological ER stress $(22,23) \cdot \mathrm{Ca}^{2+}$ has a crucial physiological role in 169 pancreatic islets as glucose-dependent cytosolic $\mathrm{Ca}^{2+}$ oscillations driven by 170 membrane electrical activity trigger insulin exocytosis (24-26) and Insulin gene 171 transcription is positively regulated by cytosolic $\mathrm{Ca}^{2+}$ levels $(27,28)$. We therefore 172 tested whether the Chop deletion-dependent changes in islets were associated 173 with altered $\mathrm{ER} \mathrm{Ca}^{2+}$ signaling, representing a logical outcome from reduced ER 174 stress (Fig. 3).

175 For this purpose, we tested the effect of a membrane-permeable intracellular $176 \mathrm{Ca}^{2+}$-chelator, BAPTA-AM (abbreviated as "BAPTA" hereafter). When islets 177 isolated from C57BL/6 mice were exposed overnight to BAPTA $(10 \mu \mathrm{M})$, we 178 observed reductions in both Ins1 and Ins2 mRNA (Fig. 4A, "Insulin" panel, Ctrl 179 versus BAPTA). At the same time, Chop and sXbp1 levels were also reduced by 180 BAPTA treatment, suggesting that lowering $\beta$-cell $\mathrm{Ca}^{2+}$ reduced UPR induction in 181 these WT islets, possibly by decreasing the Prolns translation burden placed on 182 the ER (Fig. 4A, "Chop" and "Xbp1" panel, Ctrl columns versus BAPTA-AM 
183 columns). Importantly, tunicamycin ( $\mathrm{Tm}, 0.5 \mu \mathrm{g} / \mathrm{mL})$ in combination with BAPTA 184 treatment rescued the decrease in both Ins1 and Ins2 mRNA levels, showing that 185 the effect of $\mathrm{Ca}^{2+}$ chelation on $\beta$-cell ER stress could be overcome by artificial 186 "ER stress" induction. Concomitantly, Chop and sXbp1 mRNAs, as well as BiP 187 mRNA were positively induced by Tm treatment (Fig. 4A, BAPTA columns versus $B A P T A+T m$ columns).

189 The positive regulation of Ins1/Ins2 transcription by ER stress, previously reported (9), was further confirmed in a separate batch of WT and Chop $\beta K O$ islets treated with $5 \mu \mathrm{g} / \mathrm{mL}$ Tm (Sppl. Fig. S9). In contrast, induction of Chop 192 mRNA by activating GCN2 using halofunginone (HFn, 50nM), a potent tRNA synthetase inhibitor $(29,30)$ to induce elF2 $\alpha$ phosphorylation, actually reduced Ins1 and Ins2 transcripts, as well as sXBP1 in spite of pronounced Atf4 and Chop 195 induction (Fig. 4A, Ctrl. Versus HFn). Under HFn treatment, Chop induction is partially attributable to the amino acid response element (AARE) sequence in the Chop promoter (31). Lastly, addition of cyclosporine A (CsA, 200nM) to islet media induced both Ins1 and Ins2 transcripts, suggesting the $\mathrm{ER} \mathrm{Ca}^{2+}$ effect on Ins1/Ins2 expression was unlikely due to a direct consequence of activation of the Calcineurin/NFAT pathway (32) by $\mathrm{Ca}^{2+}$.

201 Insulin granule secretion from mature $\beta$ cells is dependent on cytosolic $\mathrm{Ca}^{2+}$ oscillations and Chop $\beta K O$ islets exhibited reduced cytosolic $\mathrm{Ca}^{2+}$ concentration 203 in response to $11 \mathrm{mM}$ glucose (Fig. 4B, $\mathrm{p}<0.0001$ ). Interestingly, $\mathrm{ER} \mathrm{Ca}^{2+}$ 204 content was also reduced in these islets (Fig. 4C). Furthermore, GLP1conjugated antisense oligonucleotides (GLP1-ASO) demonstrated efficient gene knockdown in rodent islets (33), providing an attractive, independent strategy to 207 mediate Chop knockdown to test our hypothesis. We therefore treated mice having floxed Chop alleles (15) (on the C57BL/6 background without CreERT) with GLP1-Chop-ASO in vivo (Fig. 4D, GLP1-Chop-ASO), by subcutaneous

210 injections in young mice of both sexes $(0.5 \mathrm{nMole} / \mathrm{g}$ body weight, twice over 5 211 days, in the back of neck), with GLP1-conjugated control ASO (sequence not 212 homologous to any known gene in the murine genome). As expected, GLP1- 
213 Chop-ASO administration was well tolerated by the mice and specifically reduced 214 islet Chop transcript by $>60 \%$ (Fig. 4E), with minimal effects on other C/EBP 215 family members (i.e. Cebpa, Cebpb and Cebpg) in either islets or liver tissues, 216 demonstrating $\beta$ cell selectivity as reported (33). Further supporting our 217 hypothesis, treatment with GLP1-Chop-ASO reduced the ER $\mathrm{Ca}^{2+}$ pool without 218 secondarily affecting the cytosolic $\mathrm{Ca}^{2+}$ levels stimulated by $11 \mathrm{mM}$ glucose 219 (compare Fig. 4F and 4G), suggesting the change in $\mathrm{ER} \mathrm{Ca}^{2+}$ was a primary 220 event that immediately followed Chop knockdown. To our knowledge, this is the 221 first proof-of-principle that GLP1-ASO strategy can be exploited to alter islet 222 physiology and $\mathrm{Ca}^{2+}$ dynamics. These results support our working hypothesis 223 featuring an ER-centric role of CHOP in pancreatic $\beta$ cells (Fig. 3H, Model).

224 In summary, we propose that CHOP is activated by UPR signaling through the 225 PERK branch, as a response to increased Prolns synthesis and misfolding (34). 226 In turn, CHOP serves as a transcriptional hub to maintain ER proteostasis. This 227 process also controls Insulin transcription, partially via $\mathrm{Ca}^{2+}$ signaling, from the 228 ER to the nucleus, although this is unlikely directly mediated by CHOP (Fig. $\mathbf{3 H}$, 229 indicated by the blue arrow on right). Furthermore, ER remodeling in turn has a 230 profound effect on $\mathrm{ER} \mathrm{Ca}^{2+}$ that subsequently contributes to controlling the 231 increase in cytosolic $\mathrm{Ca}^{2+}$ that occurs in response to elevated glucose (Fig. $\mathbf{3 H}$, 232 as indicated by the blue arrow on left). At the same time, comparison of genetic 233 Chop deletion model versus the GLP1-ASO mediated Chop knockdown model 234 demonstrated that Chop deletion induced GSIS change is a long term complex 235 event in $\beta$ cells, with the $\mathrm{ER} \mathrm{Ca}^{2+}$ pool change preceding insulin mRNA reduction 236 and GSIS decrease.

237 As the pancreatic islet is a nutrient sensing organ, and $\beta$-cells secrete insulin to 238 increase anabolic metabolism upon nutrient availability, we speculate that when 239 there is a surfeit of nutrition, increased insulin secreted into the circulation 240 exacerbates IR and promotes fatty liver in humans (7), although there was a 241 dissociation between HOMA-IR (17) and hyperinsulinemia in Chop $\beta K O$ mice 242 (Fig. S1B and S3B). We were initially intrigued to find that a $75 \%$ reduction in 
243 Insulin mRNA by Chop deletion did not produce a strong metabolic phenotype in

244 mice, although this was not unprecedented $(5,6,11)$. Insulin mRNA may be in

245 excess in $\beta$-cells and crucial for glucose sensing. This may be better explained

246 by a model (Fig. $3 \mathrm{H}$ ) proposing that ER "stress" due to increased proinsulin

247 synthesis is coupled to insulin secretion, mediated through an "ER $\mathrm{Ca}^{2+"}$

248 response in $\beta$-cells. In the UPR signaling cascade, however, Perk deletion (35),

249 elF2 $\alpha$ phosphorylation site mutation (36, 37), Ire1 $\alpha$ deletion (9), sXbp1 deletion

250 (38) and Atf6 $\alpha$ deletion (39) all caused deleterious outcomes in $\beta$-cells and were

251 thus unsuitable therapeutic targets. Uniquely, Chop deletion may be the only

252 example that can safely reduce "ER stress" in $\beta$-cells (12), by exemplifying a

253 "thrifty gene" providing an evolutionary advantage during famine (40). This

254 hypothesis is more attractive given our finding that $\beta$-cell specific Chop deletion

255 prevented HFD-induced hepatic steatosis (Fig. 1H), echoing recent human

256 findings ( 7 ). Inspired by the unique phenotype of Chop-deleted $\beta$-cells, we

257 discovered that a GLP1-conjugated Chop ASO could partially recapitulate "ER

258 remodeling" characterized by a reduced $\mathrm{ER} \mathrm{Ca}^{2+}$ pool, thereby providing a

259 promising new therapeutic strategy for further pharmacological characterization

260 and refinement to combat human T2D and fatty liver disease.

261 (3061 words) 


\section{Acknowledgements:}

264 The generation of a Ddit3 gene floxed mouse model was a collaborative effort

265 with Dr. Ira Tabas at Columbia University. Drs. Jian-Liang Li, Feng Qi and Jun

266 Yin at SBP Applied Bioinformatics core provided guidance and helpful discussion

267 on the transcriptomic data analysis. Ms. Guillermina Garcia at the SBP Histology

268 Core facility provided technical assistance and helpful discussion on histology

269 quantification methodology using the Aperio Scanscope $F L^{\circledR}$ instrument. Tissue

270 analysis was facilitated by the SBP Histology core at Lake Nona, with technical

271 assistance from Mr. John Shelley.

272 R.J.K. is supported by NIH grants R01DK113171, R24DK110973, R37DK042394, 273 R01CA198103 and the SBP NCI Cancer Center Grant P30 CA030199. R.J.K. is

274 a member of the UCSD DRC (P30 DK063491) and an Adjunct Professor in the 275 Department of Pharmacology, UCSD. L.S.S. is supported by NIH grant 276 R01DK46409. C.L. is a member of the UCSD DRC and is supported by the NIH 277 training grant T32DK007494. V.S.P. acknowledges support from an Upjohn 278 Foundation postdoctoral fellowship. 


\section{References}

281 1. C. f. D. C. a. Prevention., National Diabetes Statistics Report, 2017. . (2017,

2. C. Weyer et al., Hypoadiponectinemia in obesity and type 2 diabetes: close association with insulin resistance and hyperinsulinemia. $J$ Clin Endocrinol Metab 86, 1930 (May, 2001).

3. S. Chon, J. F. Gautier, An Update on the Effect of Incretin-Based Therapies on beta-Cell Function and Mass. Diabetes Metab J 40, 99 (Apr, 2016).

4. C. Weyer, R. L. Hanson, P. A. Tataranni, C. Bogardus, R. E. Pratley, A high fasting plasma insulin concentration predicts type 2 diabetes independent of insulin resistance: evidence for a pathogenic role of relative hyperinsulinemia. Diabetes 49, 2094 (Dec, 2000).

5. A. E. Mehran et al., Hyperinsulinemia drives diet-induced obesity independently of brain insulin production. Cell Metab 16, 723 (Dec 5, 2012).

6. N. M. Templeman, S. M. Clee, J. D. Johnson, Suppression of hyperinsulinaemia in growing female mice provides long-term protection against obesity. Diabetologia 58, 2392 (Oct, 2015).

7. G. I. Smith et al., Insulin resistance drives hepatic de novo lipogenesis in nonalcoholic fatty liver disease. J Clin Invest, (2019).

8. M. M. Page et al., Reducing insulin via conditional partial gene ablation in adults reverses diet-induced weight gain. The FASEB Journal 32, 1196 (2018/03/01, 2018).

9. J. R. Hassler et al., The IRE1alpha/XBP1s Pathway Is Essential for the Glucose Response and Protection of beta Cells. PLoS Biol 13, e1002277 (Oct, 2015).

10. B. Kutlu et al., Detailed transcriptome atlas of the pancreatic beta cell. BMC Med Genomics 2, 3 (Jan 15, 2009).

11. M. Szabat et al., Reduced Insulin Production Relieves Endoplasmic Reticulum Stress and Induces beta Cell Proliferation. Cell Metab, (Nov 24, 2015).

12. B. Song, D. Scheuner, D. Ron, S. Pennathur, R. J. Kaufman, Chop deletion reduces oxidative stress, improves $\beta$ cell function, and promotes cell survival in multiple mouse models of diabetes. J Clin Invest 118, 3378 (2008).

13. M. Maris et al., Deletion of C/EBP homologous protein (Chop) in C57BI/6 mice dissociates obesity from insulin resistance. Diabetologia 55, 1167 (Apr, 2012).

14. T. Satoh et al., CHOP deletion does not impact the development of diabetes but suppresses the early production of insulin autoantibody in the NOD mouse. Apoptosis 16, 438 (Apr, 2011).

15. A. X. Zhou et al., C/EBP-Homologous Protein (CHOP) in Vascular Smooth Muscle Cells Regulates Their Proliferation in Aortic Explants and Atherosclerotic Lesions. Circ Res 116, 1736 (May 22, 2015).

16. Y. Dor, J. Brown, O. I. Martinez, D. A. Melton, Adult pancreatic beta-cells are formed by self-duplication rather than stem-cell differentiation. Nature 429, 41 (May 6, 2004).

17. Brian W. Parks et al., Genetic Architecture of Insulin Resistance in the Mouse. Cell Metab 21, 334 (2015).

18. J. Han et al., ER-stress-induced transcriptional regulation increases protein synthesis leading to cell death. Nat Cell Biol 15, 481 (May, 2013).

19. D. Scheuner et al., Control of mRNA translation preserves endoplasmic reticulum function in beta cells and maintains glucose homeostasis. Nat Med 11, 757 (Jul, 2005). 
20. M. Liu, Y. Li, D. Cavener, P. Arvan, Proinsulin disulfide maturation and misfolding in the endoplasmic reticulum. J Biol Chem 280, 13209 (Apr 8, 2005).

21. J. Fan et al., CTAGE5 deletion in pancreatic beta cells impairs proinsulin trafficking and insulin biogenesis in mice. J Cell Biol 216, 4153 (Dec 4, 2017).

22. R. J. Kaufman, J. D. Malhotra, Calcium trafficking integrates endoplasmic reticulum function with mitochondrial bioenergetics. Biochimica et biophysica acta 1843, 2233 (Oct, 2014).

23. J. Yong et al., Mitochondria supply ATP to the ER through a mechanism antagonized by cytosolic $\mathrm{Ca}(2)$. Elife 8, (Sep 9, 2019).

24. P. Gilon, H. Y. Chae, G. A. Rutter, M. A. Ravier, Calcium signaling in pancreatic beta-cells in health and in Type 2 diabetes. Cell Calcium 56, 340 (Nov, 2014).

25. J. Sabourin et al., Store-operated Ca2+ Entry Mediated by Orai1 and TRPC1 Participates to Insulin Secretion in Rat beta-Cells. J Biol Chem 290, 30530 (Dec 18, 2015).

26. L. S. Satin, P. C. Butler, J. Ha, A. S. Sherman, Pulsatile insulin secretion, impaired glucose tolerance and type 2 diabetes. Mol Aspects Med 42, 61 (Apr, 2015).

27. M. S. German, L. G. Moss, W. J. Rutter, Regulation of insulin gene expression by glucose and calcium in transfected primary islet cultures. J Biol Chem 265, 22063 (Dec 25, 1990).

28. M. C. Lawrence, H. S. Bhatt, J. M. Watterson, R. A. Easom, Regulation of insulin gene transcription by a $\mathrm{Ca}(2+)$-responsive pathway involving calcineurin and nuclear factor of activated T cells. Mol Endocrinol 15, 1758 (Oct, 2001).

29. T. L. Keller et al., Halofuginone and other febrifugine derivatives inhibit prolyltRNA synthetase. Nat Chem Biol 8, 311 (Feb 12, 2012).

30. H. Zhou, L. Sun, X. L. Yang, P. Schimmel, ATP-directed capture of bioactive herbal-based medicine on human tRNA synthetase. Nature 494, 121 (Feb 7, 2013).

31. A. Bruhat et al., Amino acids control mammalian gene transcription: activating transcription factor 2 is essential for the amino acid responsiveness of the $\mathrm{CHOP}$ promoter. Mol Cell Biol 20, 7192 (Oct, 2000).

32. J. J. Heit et al., Calcineurin/NFAT signalling regulates pancreatic beta-cell growth and function. Nature 443, 345 (Sep 21, 2006).

33. C. Ammala et al., Targeted delivery of antisense oligonucleotides to pancreatic beta-cells. Sci Adv 4, eaat3386 (Oct, 2018).

34. A. Arunagiri et al., Proinsulin misfolding is an early event in the progression to type 2 diabetes. Elife 8, e44532 (2019/06/11, 2019).

35. H. P. Harding et al., Diabetes mellitus and exocrine pancreatic dysfunction in perk-/- mice reveals a role for translational control in secretory cell survival. $\mathrm{Mol}$ Cell 7, 1153 (Jun, 2001).

36. D. Scheuner et al., Translational Control Is Required for the Unfolded Protein Response and In Vivo Glucose Homeostasis. Mol Cell 7, 1165 (2001).

37. S. H. Back et al., Translation attenuation through elF2alpha phosphorylation prevents oxidative stress and maintains the differentiated state in beta cells. Cell Metab 10, 13 (Jul, 2009).

38. A. H. Lee, K. Heidtman, G. S. Hotamisligil, L. H. Glimcher, Dual and opposing roles of the unfolded protein response regulated by IRE1alpha and XBP1 in proinsulin processing and insulin secretion. Proc Natl Acad Sci U S A 108, 8885 (May 24, 2011). 
378 39. F. Engin et al., Restoration of the unfolded protein response in pancreatic beta cells protects mice against type 1 diabetes. Sci Transl Med 5, 211 ra156 (Nov 13, 2013).

40. J. V. Neel, Diabetes mellitus: a "thrifty" genotype rendered detrimental by "progress"? American journal of human genetics 14, 353 (Dec, 1962).

383 


\section{Figure Legends}

386 Figure 1. $\beta$-cell specific Chop deletion reduces pancreatic and circulating insulin 387 levels and prevents liver triglyceride accumulation in male HFD fed mice.

388 Under normal diet feeding, Chop floxed $(n=4)$ and the isogenic Chop deleted $(n=4)$ 389 littermates were tested 4mon after TAM injections. After a glucose injection $(1.5 \mathrm{mg} / \mathrm{g}$ 390 body weight, i.p.), there was no difference in glucose excursion after $30 \mathrm{~min}(\mathbf{A})$, while 391 serum insulin levels were significantly reduced after $30 \mathrm{~min}$ in Chop-deleted mice $(\mathbf{B}, \mathrm{p}<$ 392 0.05, by Bonferroni's test after RM 2-Way ANOVA). (C) The body weight of individual 393 mice correlated linearly with fasting insulin levels ( $p<0.01$ by $F$-test, $\left.r^{2}=0.75\right)$. (D) Chop 394 deleted mice displayed a non-significant decrease in cumulative weight gain at $4 \mathrm{mon}$ 395 after gene deletion ( $p=0.11$ by 2 -tailed Student's $t$-test).

396 In a separate experiment, $(\mathbf{E})$ Chop floxed $(n=3)$ and congenic control littermates $(n=5$, 397 with 4 WT animals +1 heterozygous animal) were fed high fat diet (HFD, $45 \%$ kcal from 398 fat) for 20 wks before tissue harvest. Mice received TAM injections at 10wks after HFD to 399 delete Chop gene in $\beta$-cells. Experimental scheme is shown. (F) Reduced body weight 400 gain of Chop deleted mice became significant at 20wks ( $p<0.01$, 10wks after Chop 401 deletion by TAM). (G) No significant difference was detected for non-fasting blood 402 glucose levels between the two groups. (H) Fresh liver (upper) and pancreas (lower) are 403 shown for all mice, immediately after tissue dissection. (I) Liver weight was significantly 404 less for Chop-deleted animals (Chop $\Delta / \Delta$ : Cre, after CreERT-mediated Chop deletion) 405 compared to WT and Het littermate control mice $(p=0.02)$. (J) Liver triglyceride levels 406 were significantly lower in Chop-deleted mice, compared to WT and Het littermate 407 control mice $(p<0.05)$. (K) Pancreatic insulin contents were reduced in Chop deleted 408 mice compared to littermate control mice, albeit non-significantly $(p=0.11)$. (L) Liver 409 triglyceride content correlated linearly with pancreatic insulin content on a semi-log plot $410 \quad(p<0.05)$. 
412 Figure 2. Beta-cell specific Chop deletion improves insulin secretion after

413 sustained glucose stimulation, without altering glucose metabolism.

414 Mice at $9 w k s$ of age were fed $45 \%$ HFD for $22 w k s$ and after $10 w k s$ TAM was injected.

415 Floxed Chop heterozygous littermates served as controls (for which deletion of one

416 allele was accomplished by TAM injection). There was no significant difference in body

417 weight (A), daily food intake (B), non-fasting blood glucose levels (C) or glucose

418 excursion after an IPGTT (D).

419 In a separate experiment, similar comparison groups were used in a hyperglycemic

420 clamp test: (E) shows Experimental Scheme. Chop-floxed mice with their congenic

421 littermates were fed with HFD for $7 w k s$ before TAM injection. Mice were fed HFD for

422 another $12 \mathrm{wks}$ before the hyperglycemic clamp assay ( $\mathrm{n}=3$ for Chop $+/-$ : Cre and $\mathrm{n}=8$

423 for Chop $\Delta /-$ : Cre, both male and female mice were tested). (F and G) Insulin and C-

424 peptide ELISA assays demonstrated a significant increase in Chop deleted mice after

$42540 \mathrm{~min}(\mathrm{p}=0.01)$. (H) The hyperglycemic clamp was maintained at $300 \mathrm{mg} / \mathrm{dL}$ by

426 continuous glucose infusion into the jugular vein via an implanted catheter. (I) Glucose

427 infusion rates (GIR) were significantly higher for $\beta$-cell specific Chop KO mice $(p=$

428 0.0001). (J) There was no difference in insulin sensitivity, defined as glucose disposition

429 rate (GDR) divided by Insulin plasma concentration, between the two groups $(p=0.82)$.

430 After the hyperglycemic clamp, pancreata were dissected and processed for histological

431 analysis. (K) Pancreatic insulin immuno-positive areas were similar in both groups.

432 For panels A to J, RM- 2 Way ANOVA was applied for statistics. Data are presented in 433 dot plot for individual mice, with Bar and Whiskers representing Mean \pm S.E.M. 
Figure 3. Chop deletion reduces Insulin transcripts and reduces ER stress in islets of male HFD fed mice.

437 The Chop floxed (Fe/Fe: Cre) and their wildtype (+/+:Cre) congenic littermates 438 ( $n=5 /$ group, all male) were fed HFD (45\% cal from fat) for $45 w k s$ before TAM injections 439 and were continued on HFD for another 8wks before islet isolation. Transcripts encoding 440 Insulin genes (Ins1 and Ins2) and UPR genes were reduced in Chop KO islets. 441 Representative qRT-PCR results from one pair shown (A, B). The same qRT-PCR 442 experiment was repeated five times using different pairs of mice, e.g. results shown in 443 Fig. S9.

444 Subsequently, islet RNA was extracted from the same donors as in A and B were further 445 analyzed by RNA-Seq ( $\mathrm{n}=3$ / group). Transcriptome profiles were performed using poly-A 446 enriched mRNA by Next-Generation Sequencing. (C) Volcano plot of mRNA differential 447 expression was generated for both groups. Genes with high fold-change and/or highest $448 \mathrm{p}$-values were labeled as green/red dots to reflect down-/up-regulation as a result of 449 Chop deletion. (D) RPKM values for important $\beta$-cell lineage genes Pdx1, Nkx6.1, Mafa, 450 Ngn3 and Is/1, were unchanged and plotted with $\mathrm{p}$-value indicated for comparison 451 between KO and WT mice. (E) RPKM values for selected UPR genes were plotted with 452 p-values indicated. (F) Heat-map was generated for a selected group of genes important 453 for ER protein synthesis/translocation, with percentage change represented by the heat 454 map. (G) Co-IP verified decreased ER stress in $\beta$-cells. BiP-bound ProlNS was 455 quantified by anti-Ins co-IP in $\beta$-cell specific Chop KO islets, 6wks after TAM injection. 456 Islets from male donors (Chop $\Delta /+$ : Cre vs. Chop $\Delta / \Delta$ : Cre, $\mathrm{n}=3 /$ group) were pooled for 457 overnight culture an then glucose challenged. Two concentrations of glucose were 458 tested as indicated. INS and ProINS proteins were IP'ed by a monoclonal mouse anti459 INS antibody (Invitron, Clone 3B1) and BiP was detected by a monoclonal rabbit anti-BiP 460 antibody (kind gift from L. Hendershot). Co-IP'ed BiP was further quantified by optical 461 density (OD). (H) Working Model: Chop induction calibrates ER stress from Prolns 462 synthesis. In turn, CHOP mediates ER remodeling to influence insulin transcription and 463 granule release, mainly through $\mathrm{ER} \mathrm{Ca}^{2+}$ signaling (indicated by green arrowheads) and 464 likely through other metabolic cues (unidentified, represented by " $X$ " and " $Y$ "). 
Figure 4. ER stress induces insulin mRNAs via increasing the releasable ER $\mathrm{Ca}^{2+}$ pool and Chop depletion reduces the ER $\mathrm{Ca}^{2+}$ pool.

468 (A) Islets isolated from male C57BI6/J $(n=5)$ were pooled and divided into five groups 469 (after overnight culture, with $\sim 120$ islets/group). The islets were subsequently challenged 470 with either DMSO (labeled as “Ctrl") or one of the following compounds: 1) 10 $\mu \mathrm{M}$

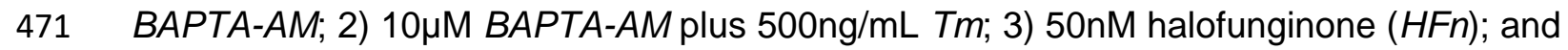
472 4) $200 \mathrm{nM}$ CsA, for $18 \mathrm{hrs}$ before RNA extraction for qRT-PCR. The relative transcript 473 abundance is summarized by bar graphs, using $\beta$-Actin expression (expressed as "Mean 474 Cq") as a reference. Both cytosolic $\mathrm{Ca}^{2+}(\mathbf{B})$ and $\mathrm{ER} \mathrm{Ca}^{2+}$ pools $(\mathbf{C})$ were reduced in $\beta$ 475 cell specific Chop KO islets. Representative traces show intracellular $\mathrm{Ca}^{2+}$ signal (mean $476 \pm 95 \% \mathrm{Cl})(\mathrm{n}=30$ - 50 islets/group). Experiments were repeated three times using three 477 pairs of control and $\beta$-cell Chop-deleted islets. Experimental conditions included 478 physiological extracellular $\mathrm{Ca}^{2+}(2.56 \mathrm{mM})$, followed by extracellular $\mathrm{Ca}^{2+}$ removal, and 479 exposure to the reversible SERCA pump inhibitor CPA $(50 \mu \mathrm{M})$ with glucose at $5 \mathrm{mM}$ 480 (“5G”) or $11 \mathrm{mM}$ (“11G”). Two-way ANOVA with post-hoc Tukey multiple comparison test 481 were used for statistical analysis. (D) Illustration shows GLP1-ASO chemical structure.

482 (E) GLP1-Chop-ASO reduces Chop mRNA (expressed as TPM) specifically in murine 483 islets compared to liver tissue. RNA extracted from the livers of the same mice served as 484 internal controls for Chop expression, for RNA-Seq. Expression of additional C/EBP 485 transcription factor family members are shown as controls. GLP1-Chop-ASO had no 486 obvious effects on glucose-induced cytosolic $\mathrm{Ca}^{2+}$ level $(\mathbf{F})$, and reduced $\mathrm{ER} \mathrm{Ca}^{2+}$ pools 487 (G) in primary islets in vivo. Representative intracellular $\mathrm{Ca}^{2+}$ traces (mean $\pm \mathrm{SEM}$ ) are 488 shown ( $\mathrm{n}=30-50$ islets/group). For panels $\mathbf{F}$ and $\mathbf{G}$, male and female Chop floxed mice 489 (with no RIP-CreER gene) were injected I.P. with control or GLP1-Chop-ASOs 8 days 490 before islet isolation. Two-way ANOVA with post-hoc Tukey multiple comparison test 491 was used for statistical analysis. 
Yong et al., Figure 1

\section{A Normal Diet}

Chop Fe/Fe : Cre

Chop NA : Cre

Blood glucose

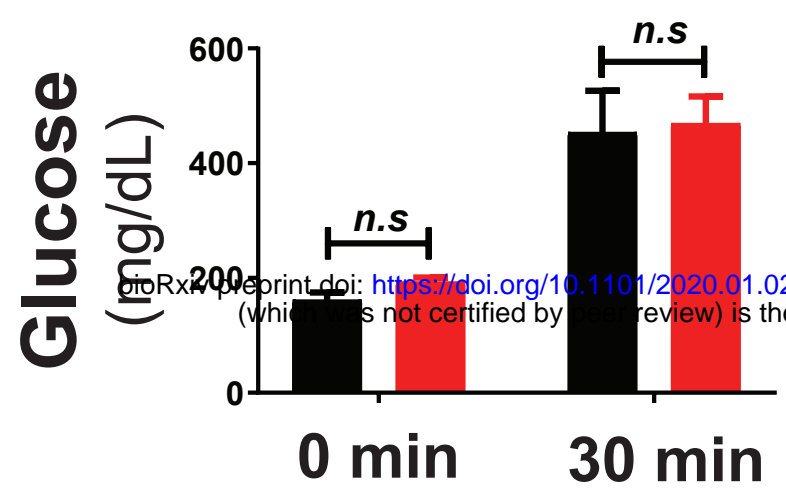

(+Glc)

B

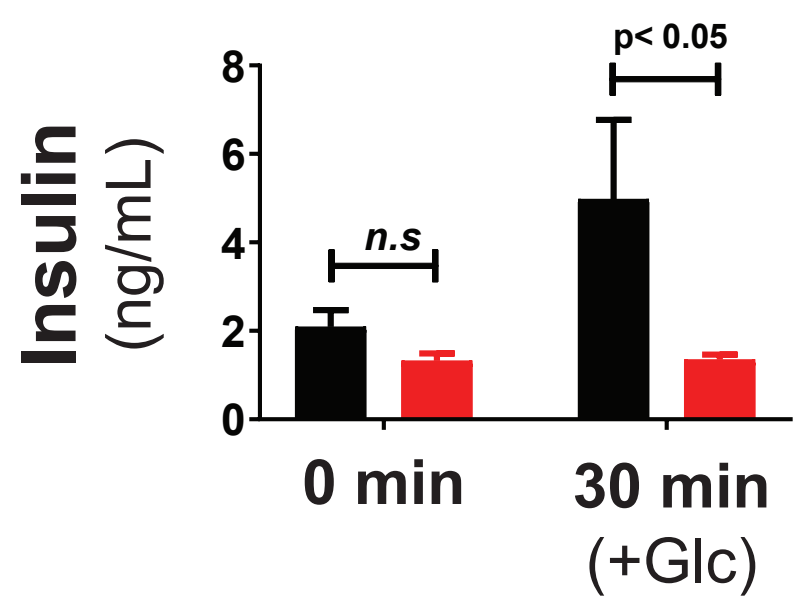

C Weight vs. Insulin

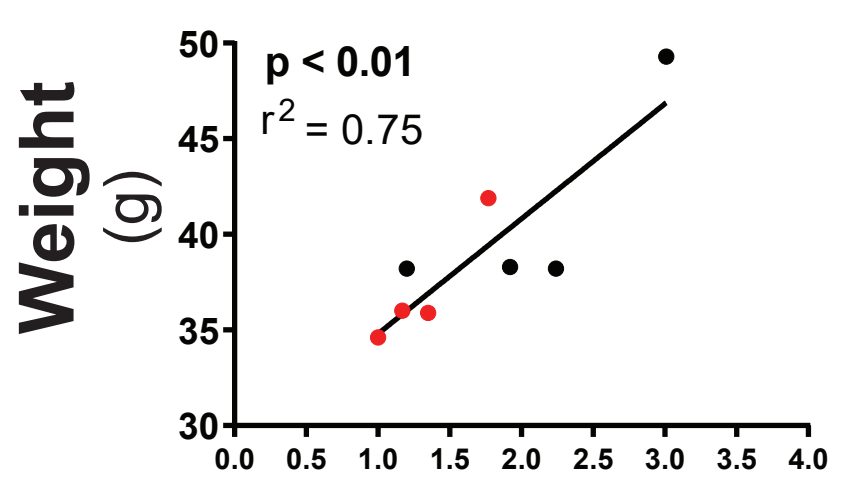

Serum Insulin

( $\mathrm{ng} / \mathrm{mL}$, fasting)

D

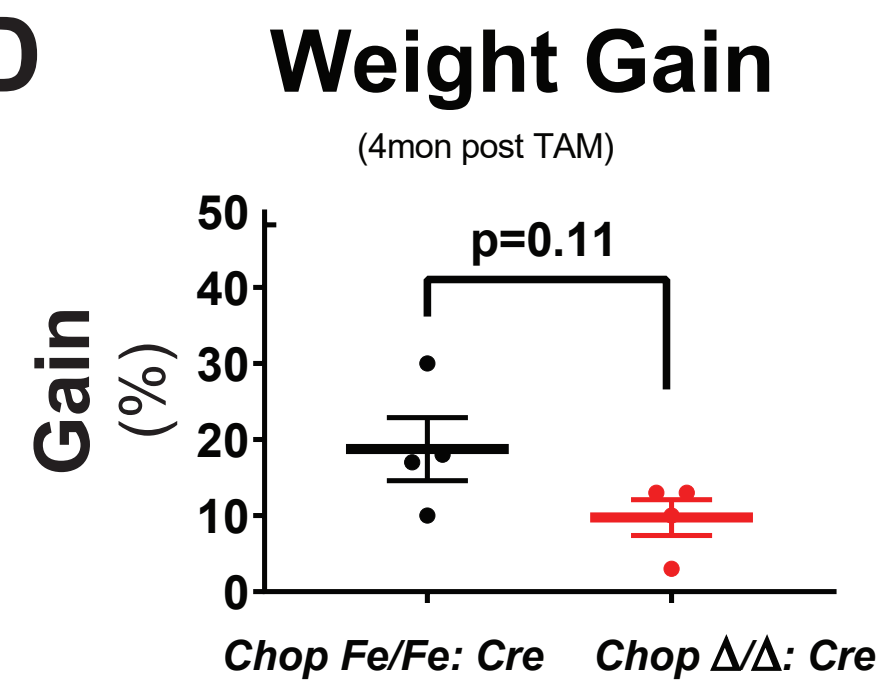

E

DIO Experimental Scheme

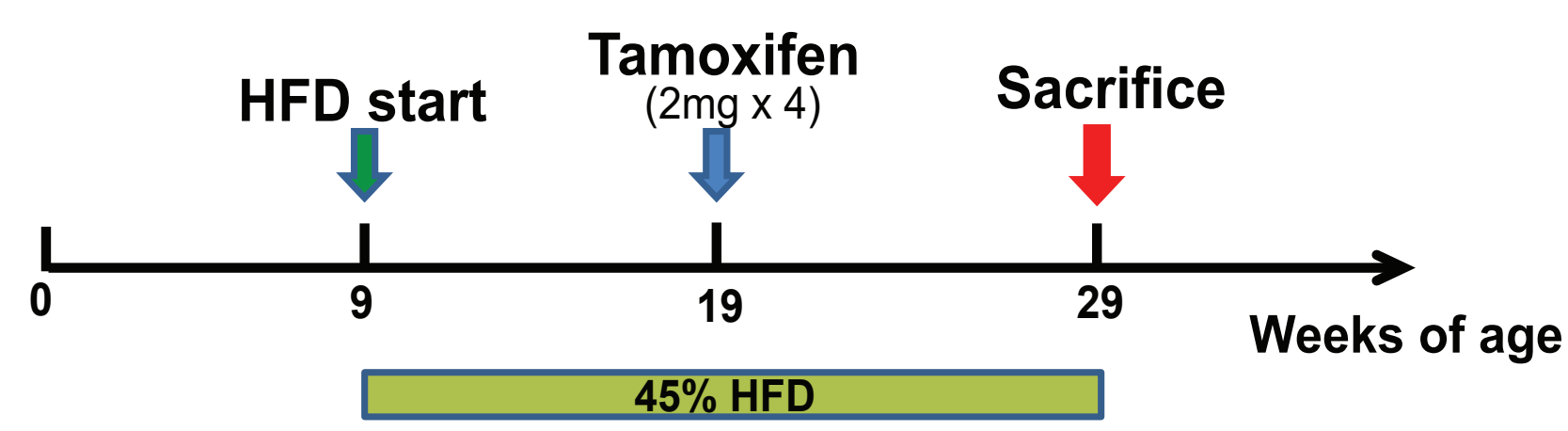

Congenic Littermates

Weight

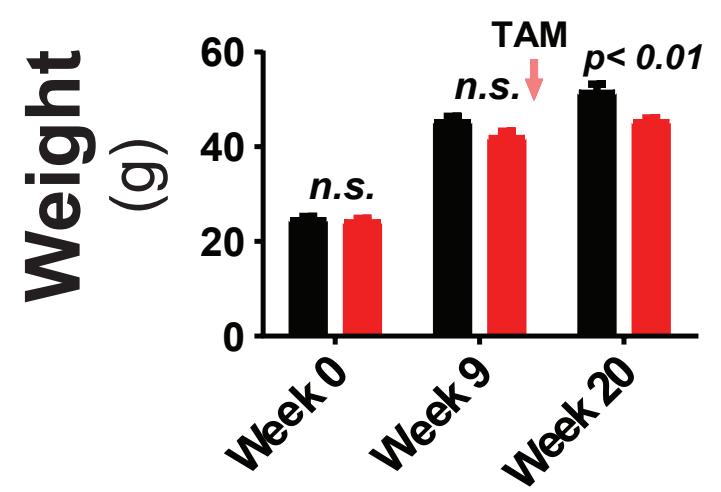

H Liver Morphology

$+/+$ : Cre
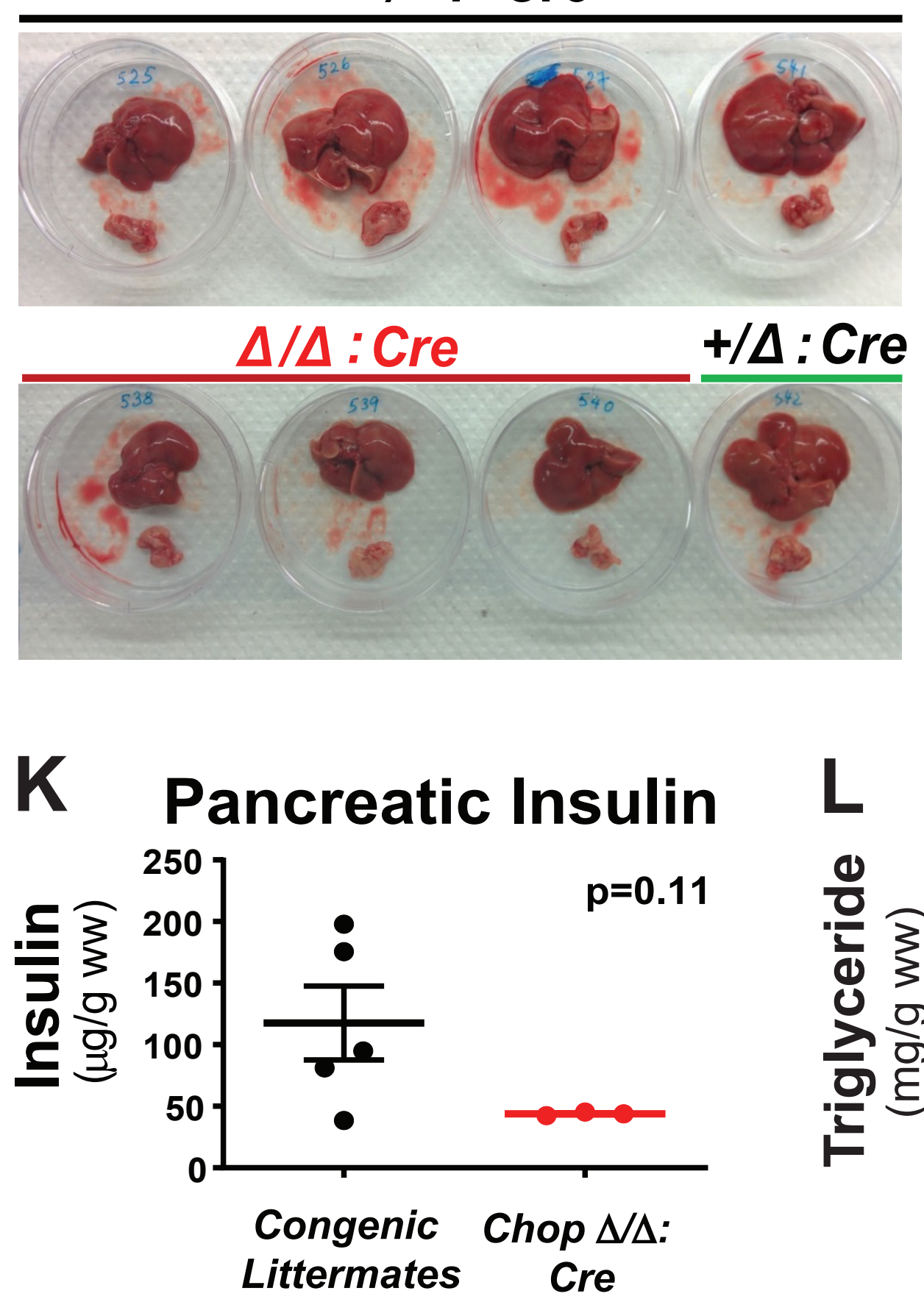

Chop Fe/Fe : Cre

G Non-fasting Glc

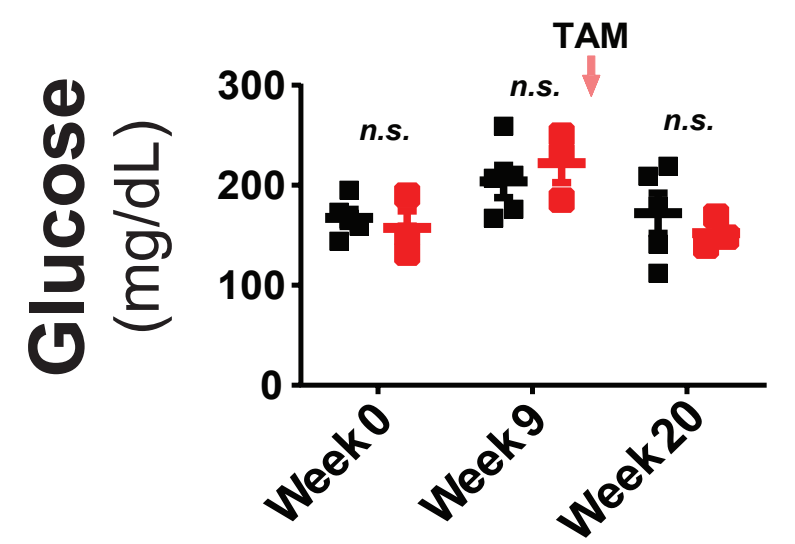

Liver Weight

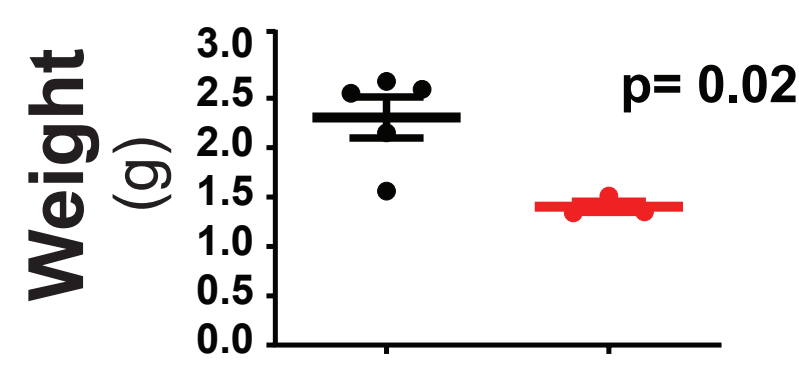

Liver TG

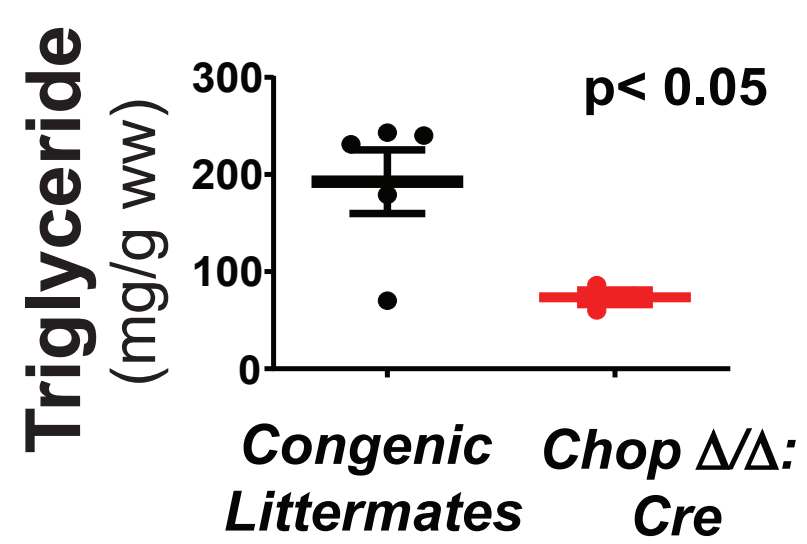

\section{K Pancreatic Insulin L Liver TG vs. Insulin ${ }^{300}$}


Yong et al., Figure 2

High fat diet (45\%)

- Chop Fe/+ : Cre

- Chop Fe/- : Cre

A

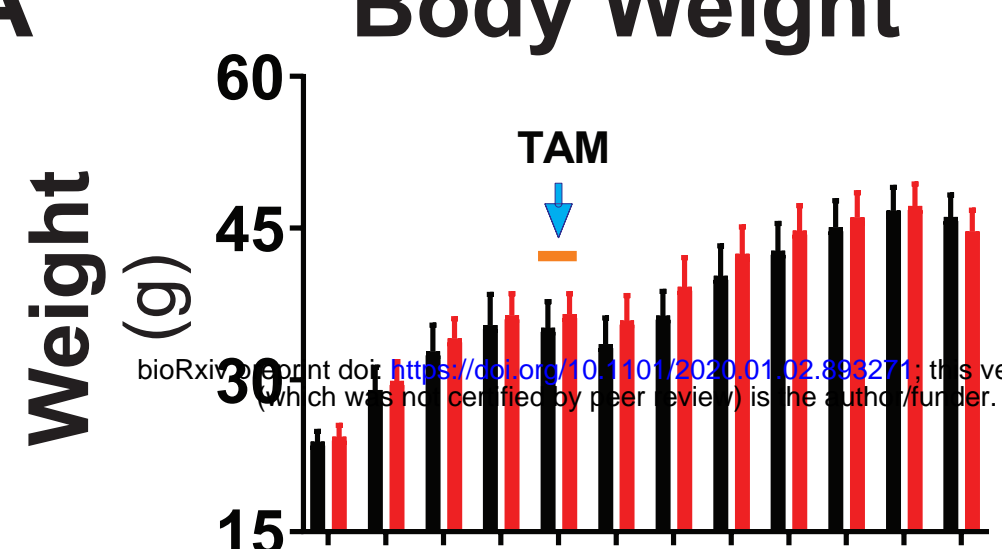

or $68,0,2,1,10,8,0,2$ Wks

Weeks post HFD

B

Food Intake

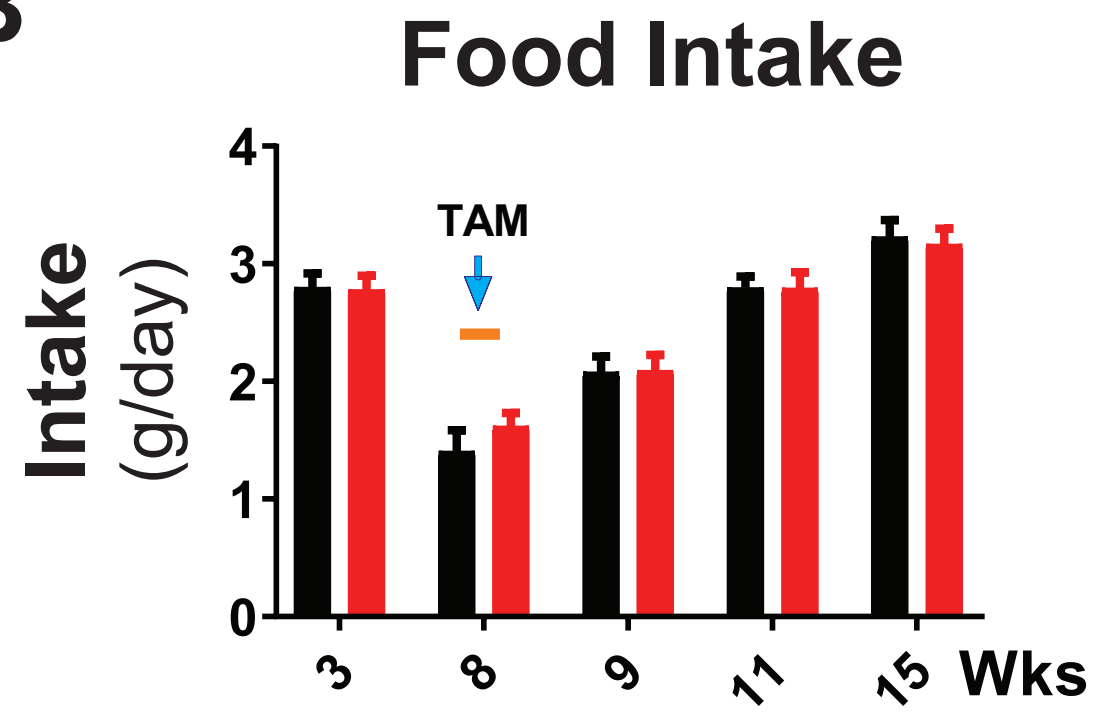

C

D

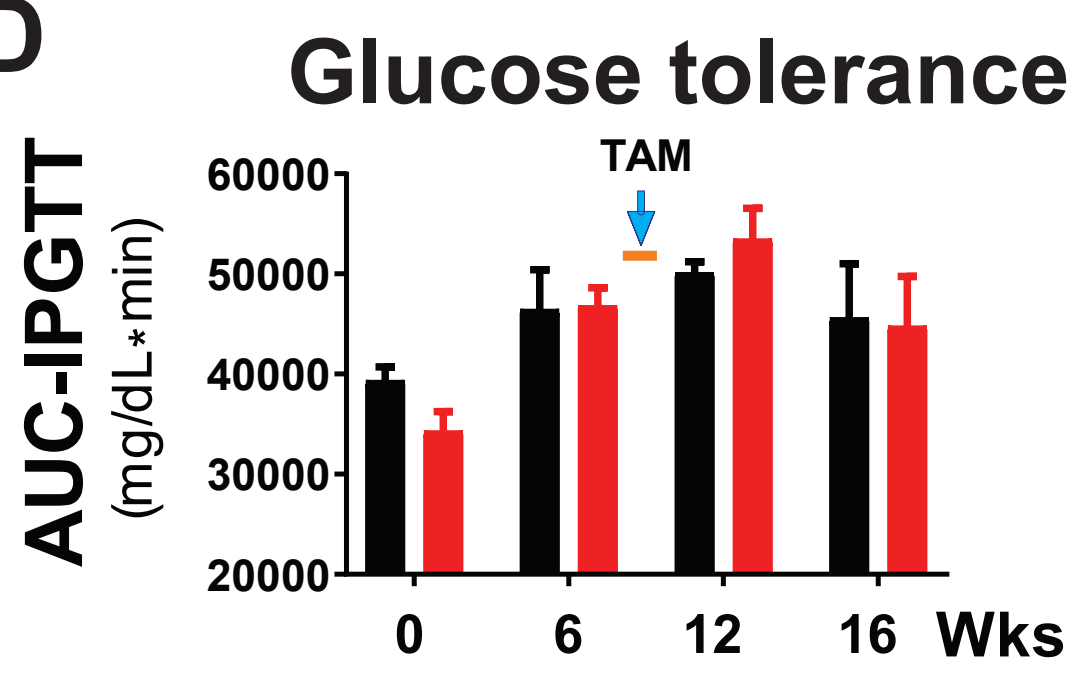

Non-fast GIc
E Clamp study on DIO mice

,

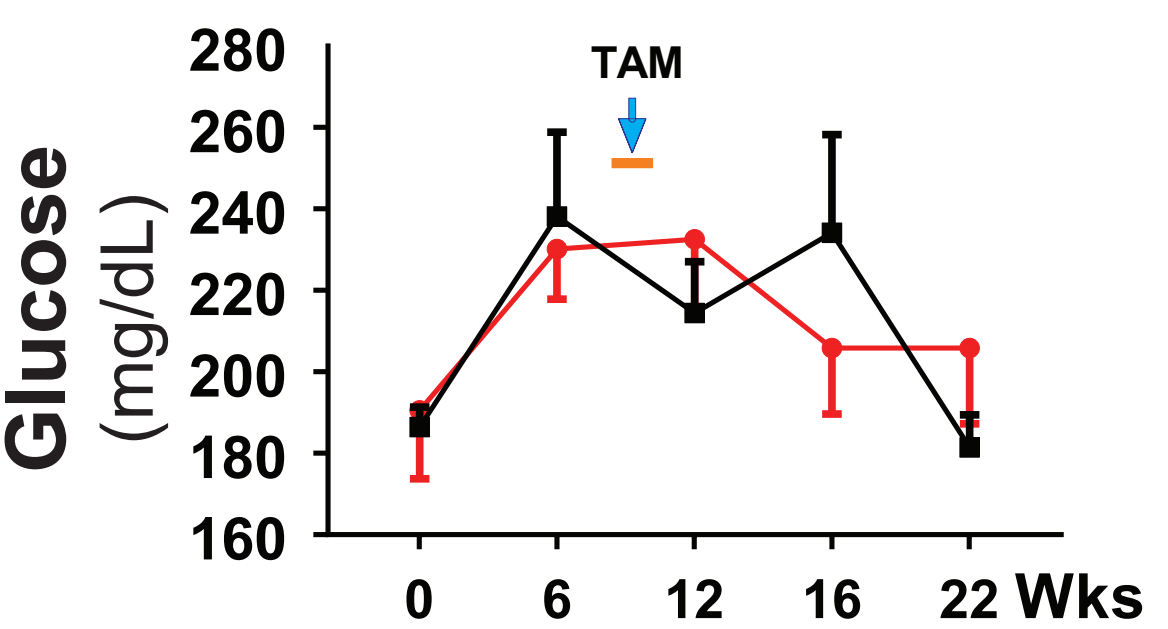

J Insulin Sensivity

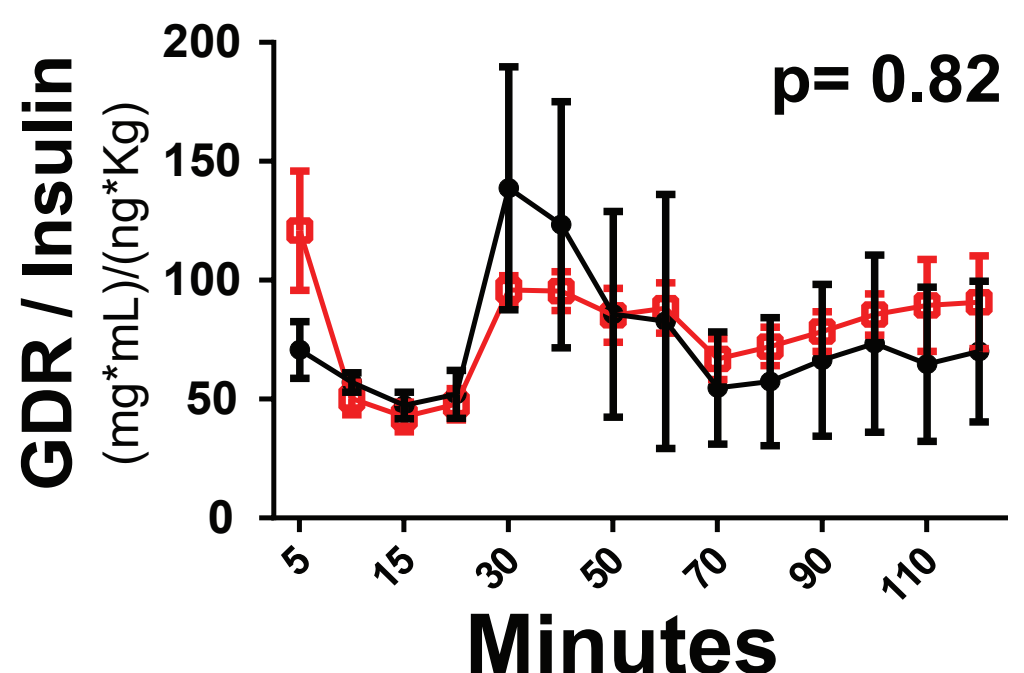

Tamoxifen Hyperglycemic $(2 \mathrm{mg} \times 4)$ clamp test

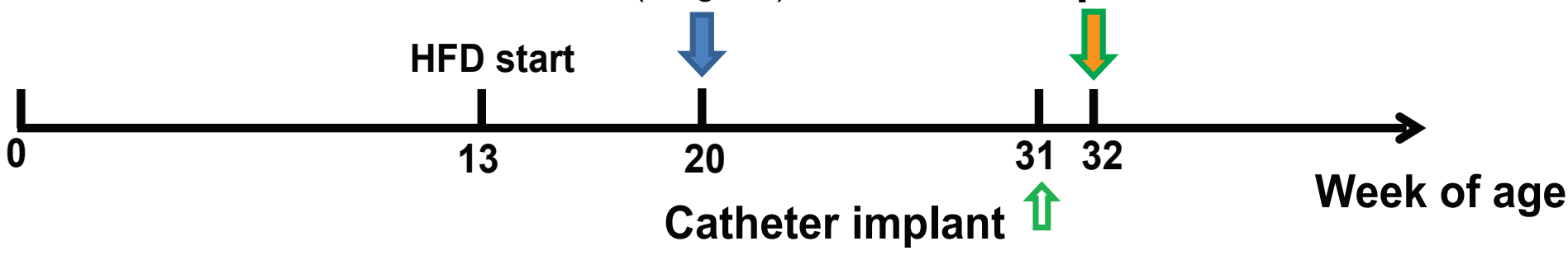

F

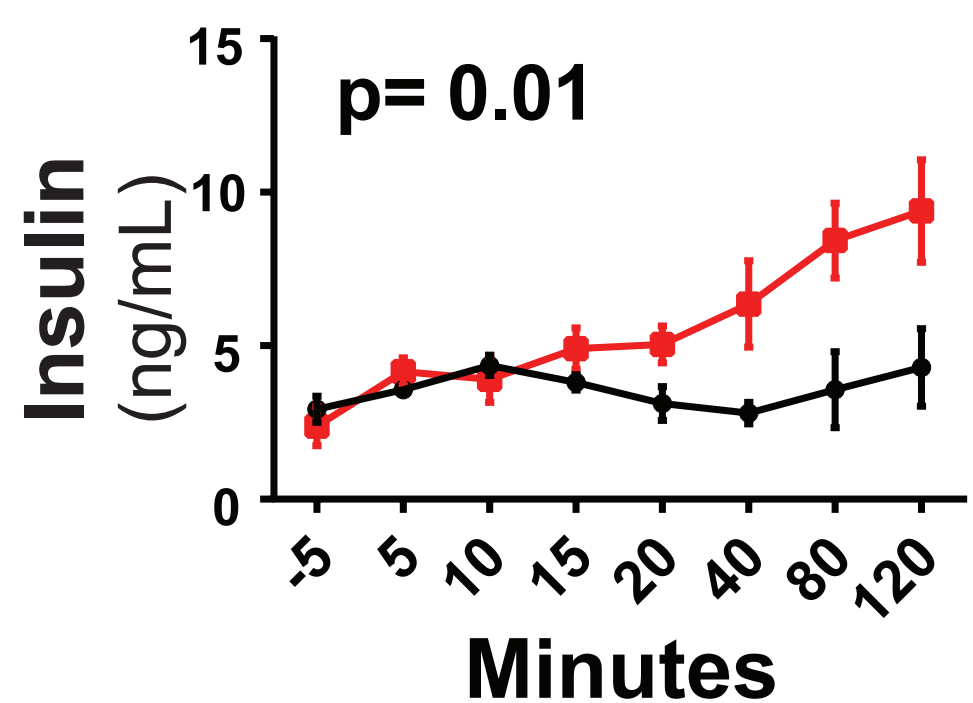

G

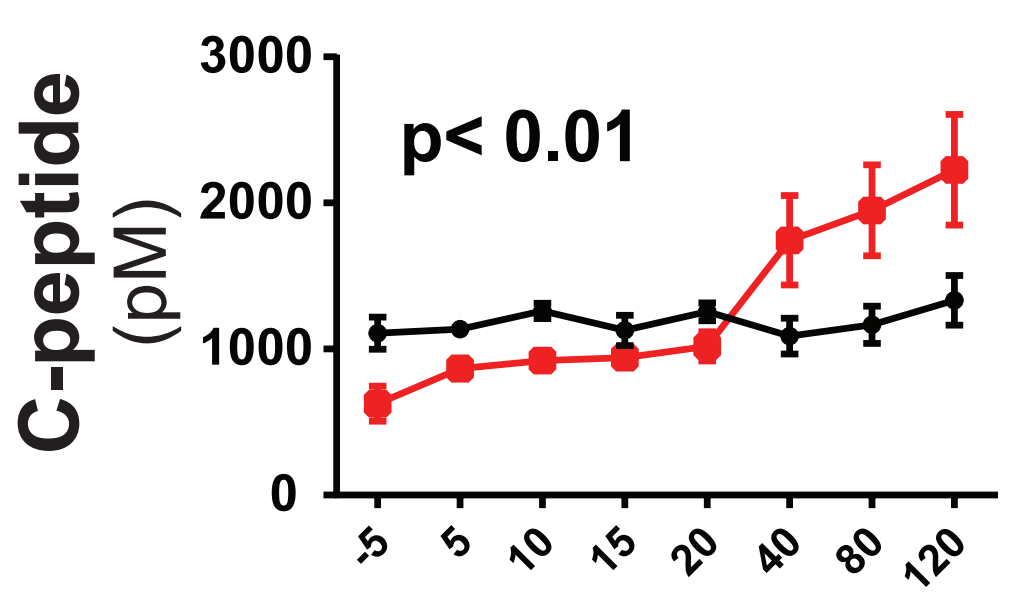

Minutes

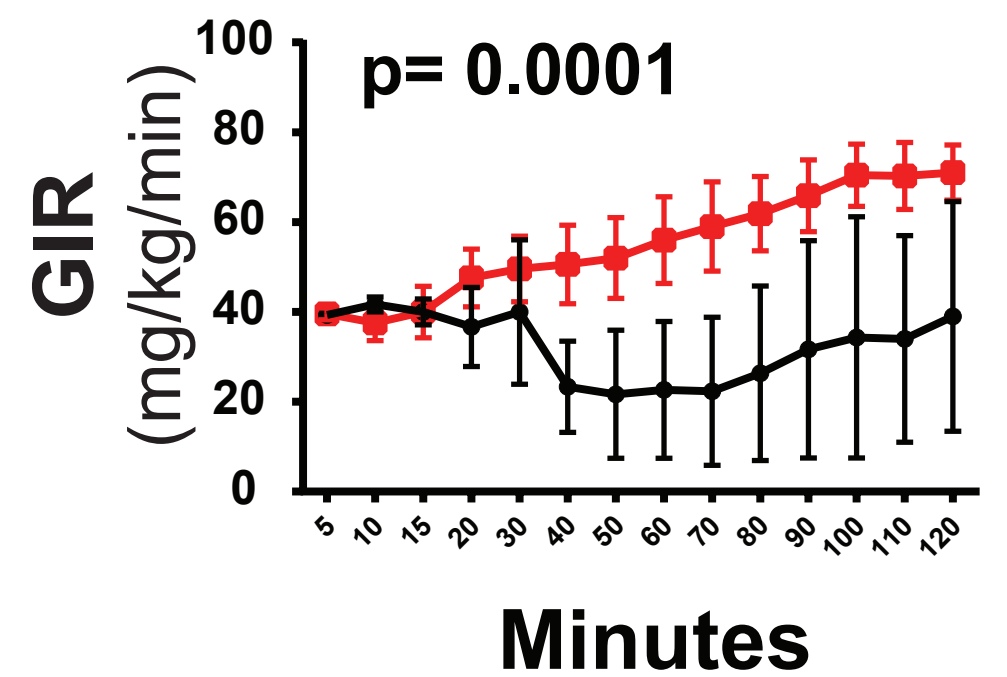

K

1

$\mathbf{H}$

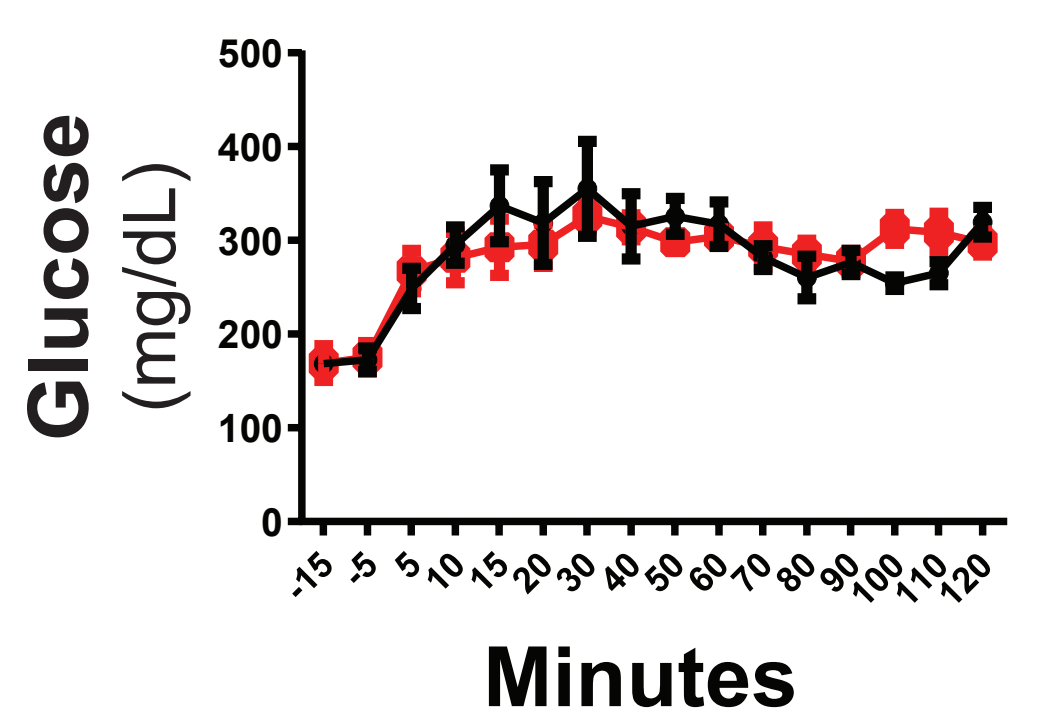

Minutes

Infusion Rate

Insulin ${ }^{+}$Area

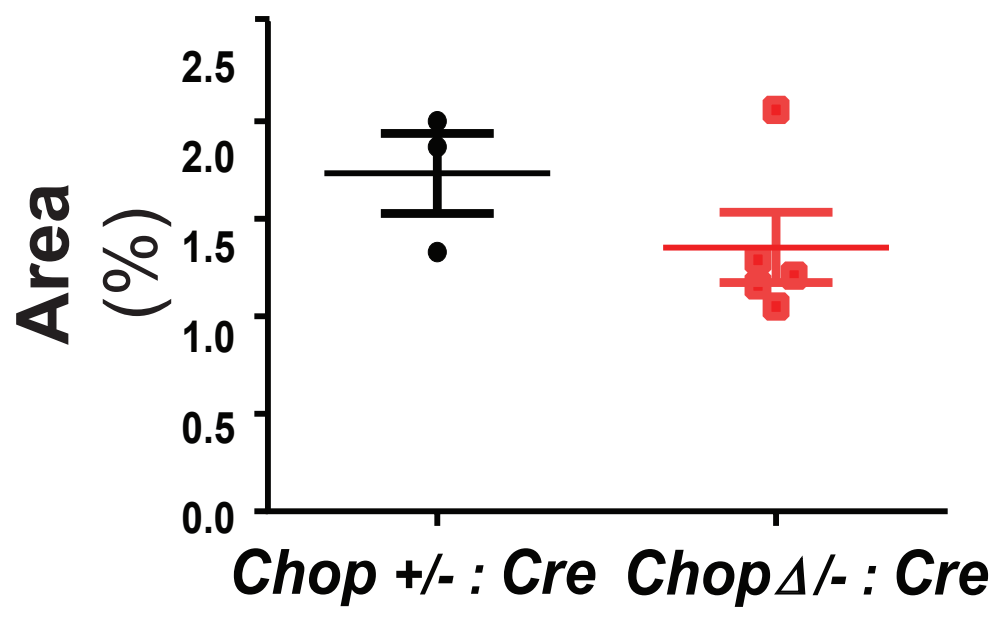


A
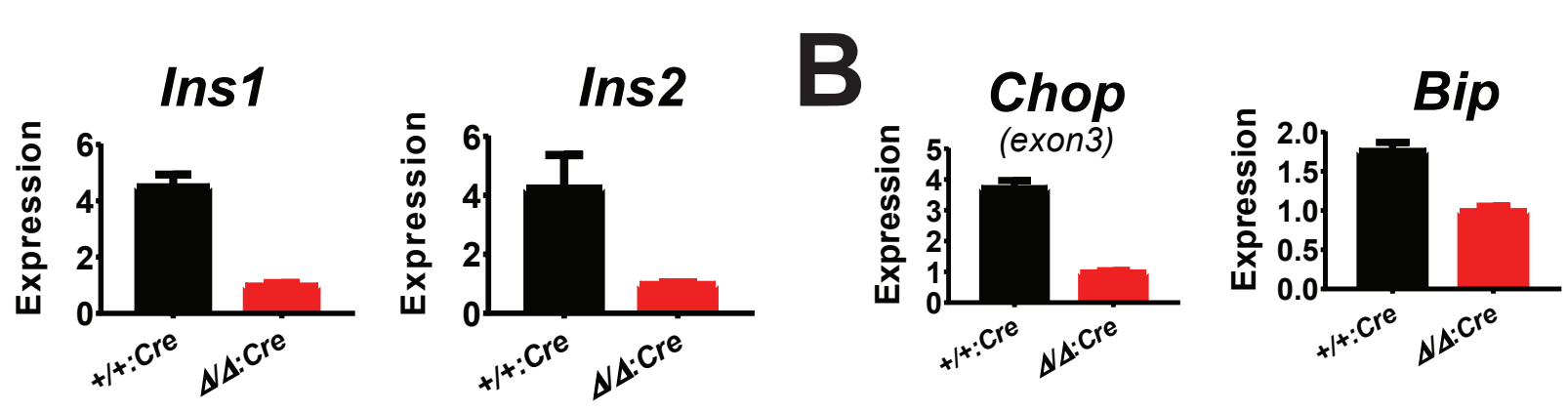

D
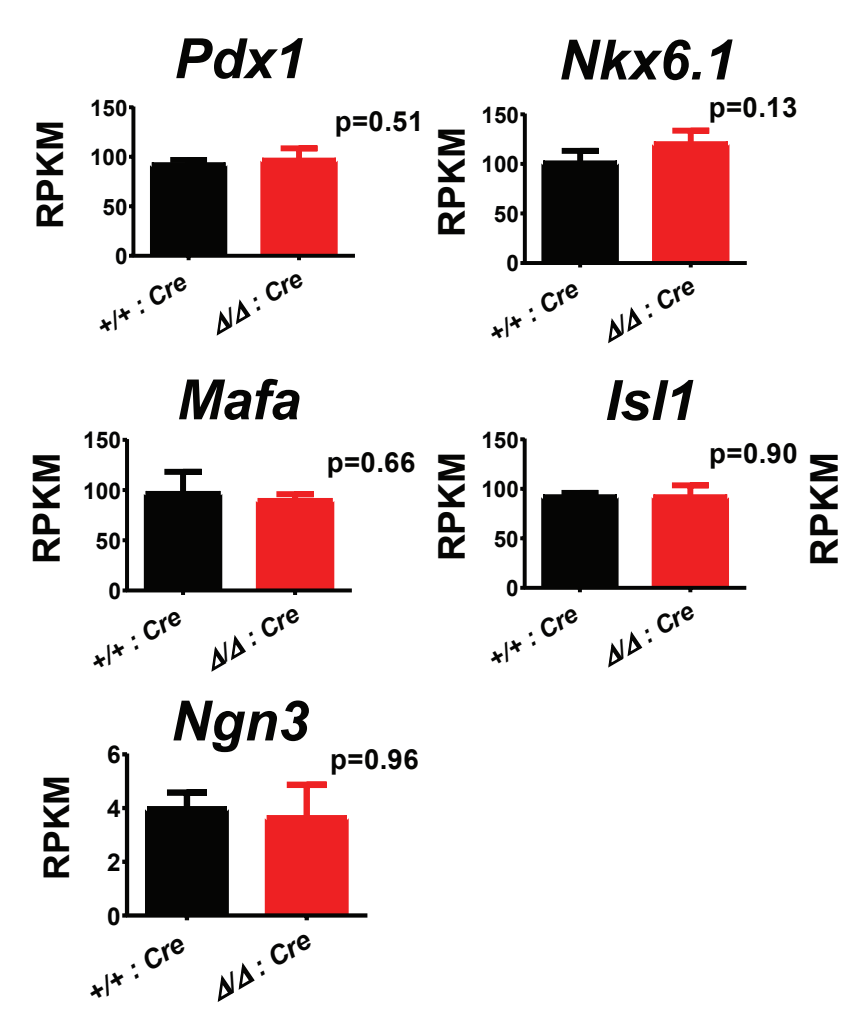

F

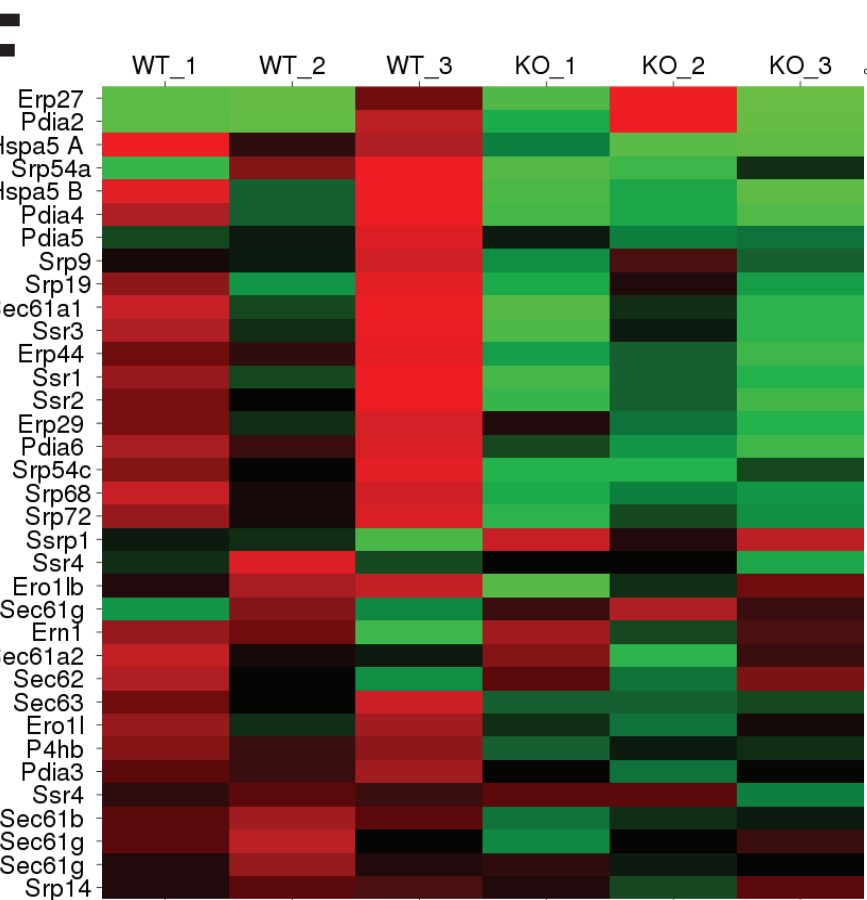

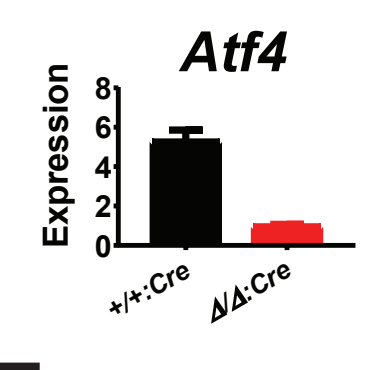

E

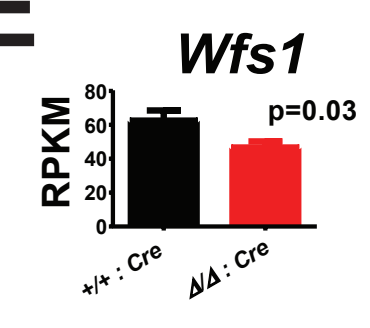

Bip

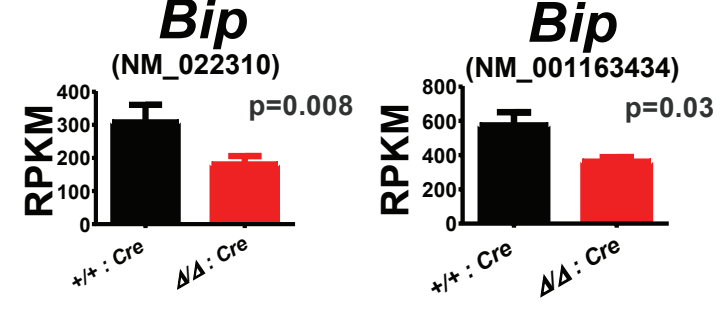

G

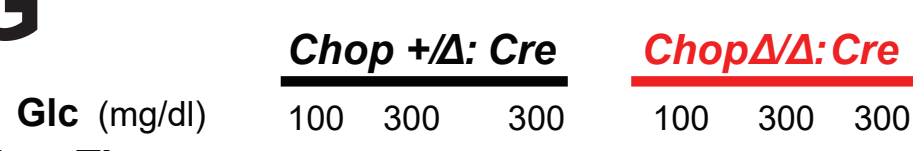
Inc. Time (min) $45 \quad 45 \quad 30 \quad 45 \quad 45 \quad 30$

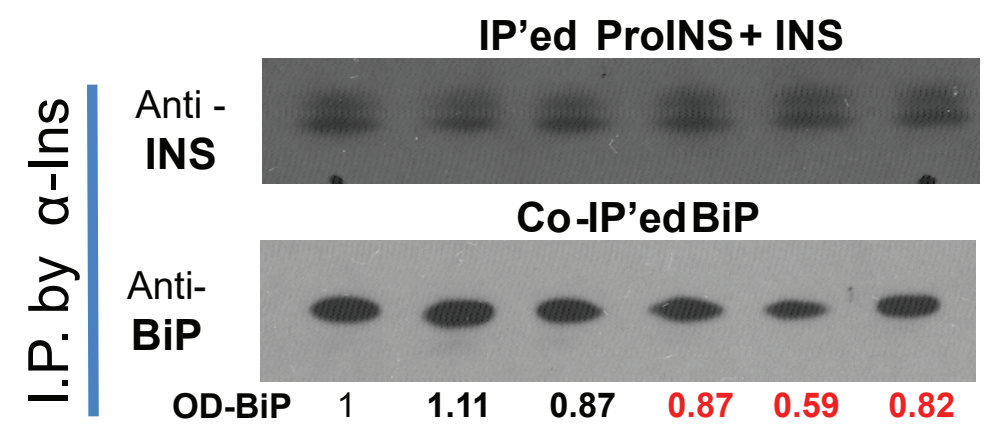

$$
\begin{aligned}
& \text { 골 Anti- } \\
& \text { ㅅctin }
\end{aligned}
$$

H
Txndc5
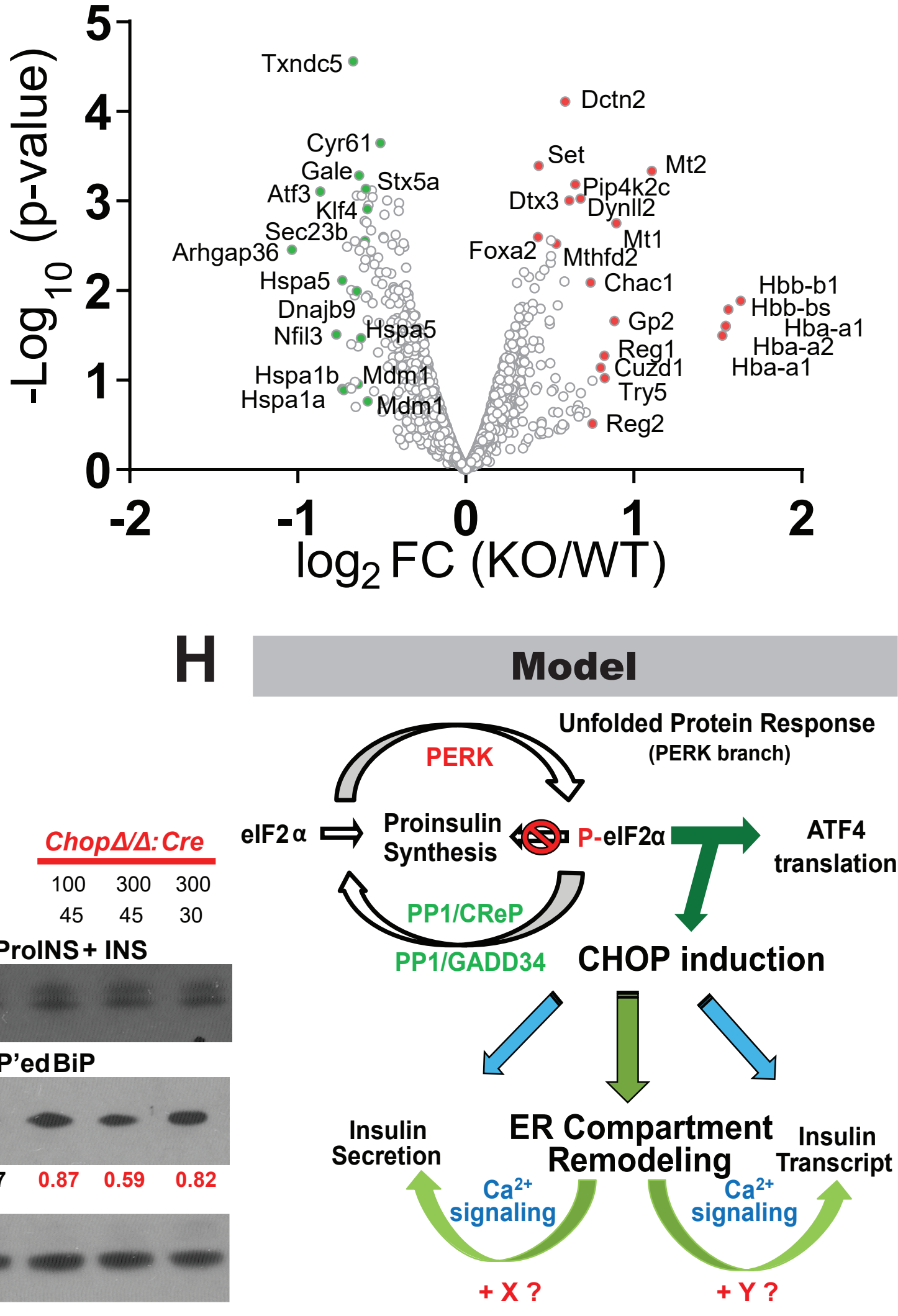
A

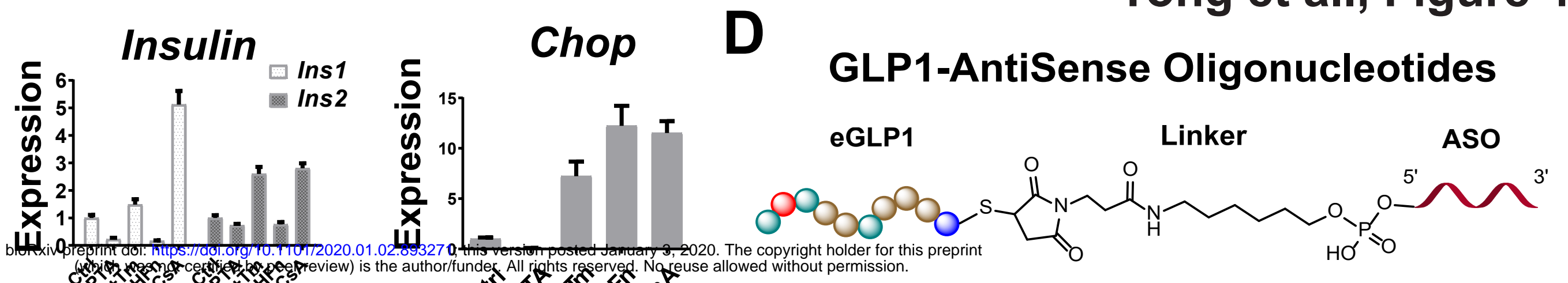

\section{GLP1-AntiSense Oligonucleotides}
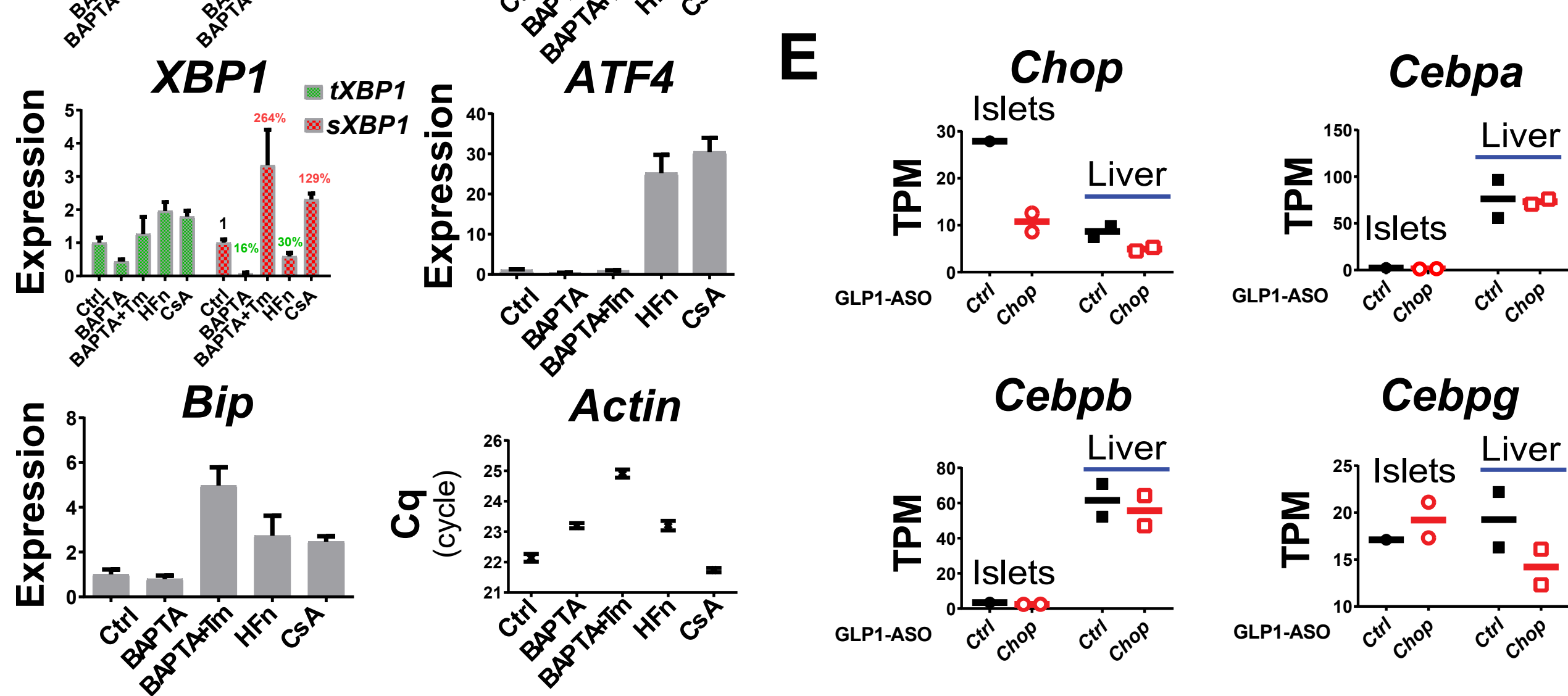

Cebpb

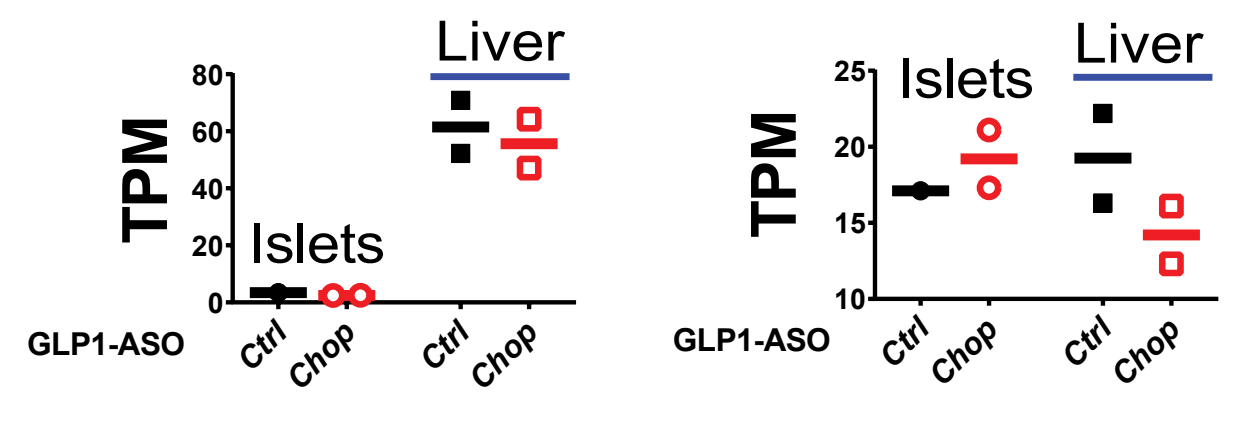

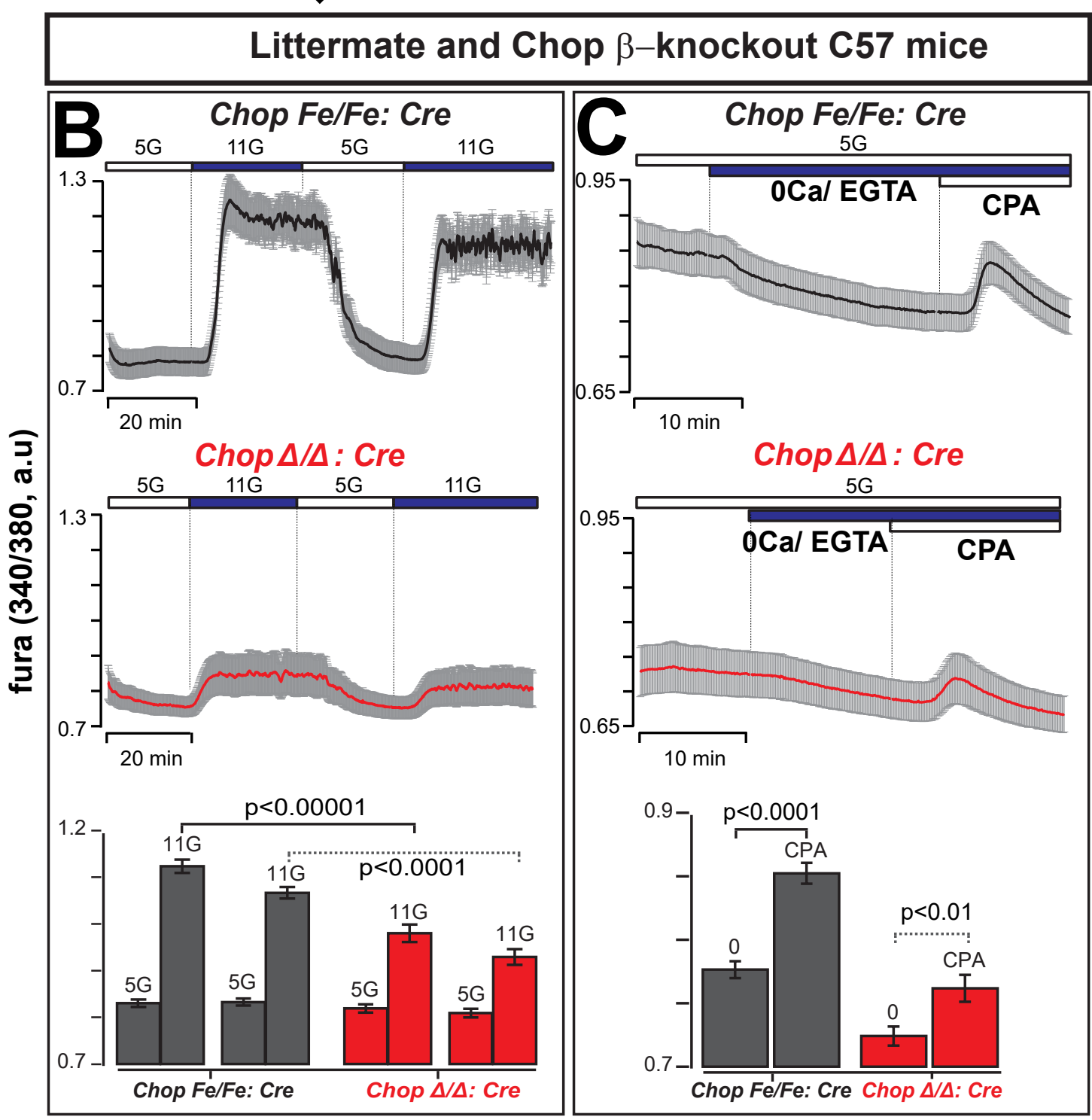

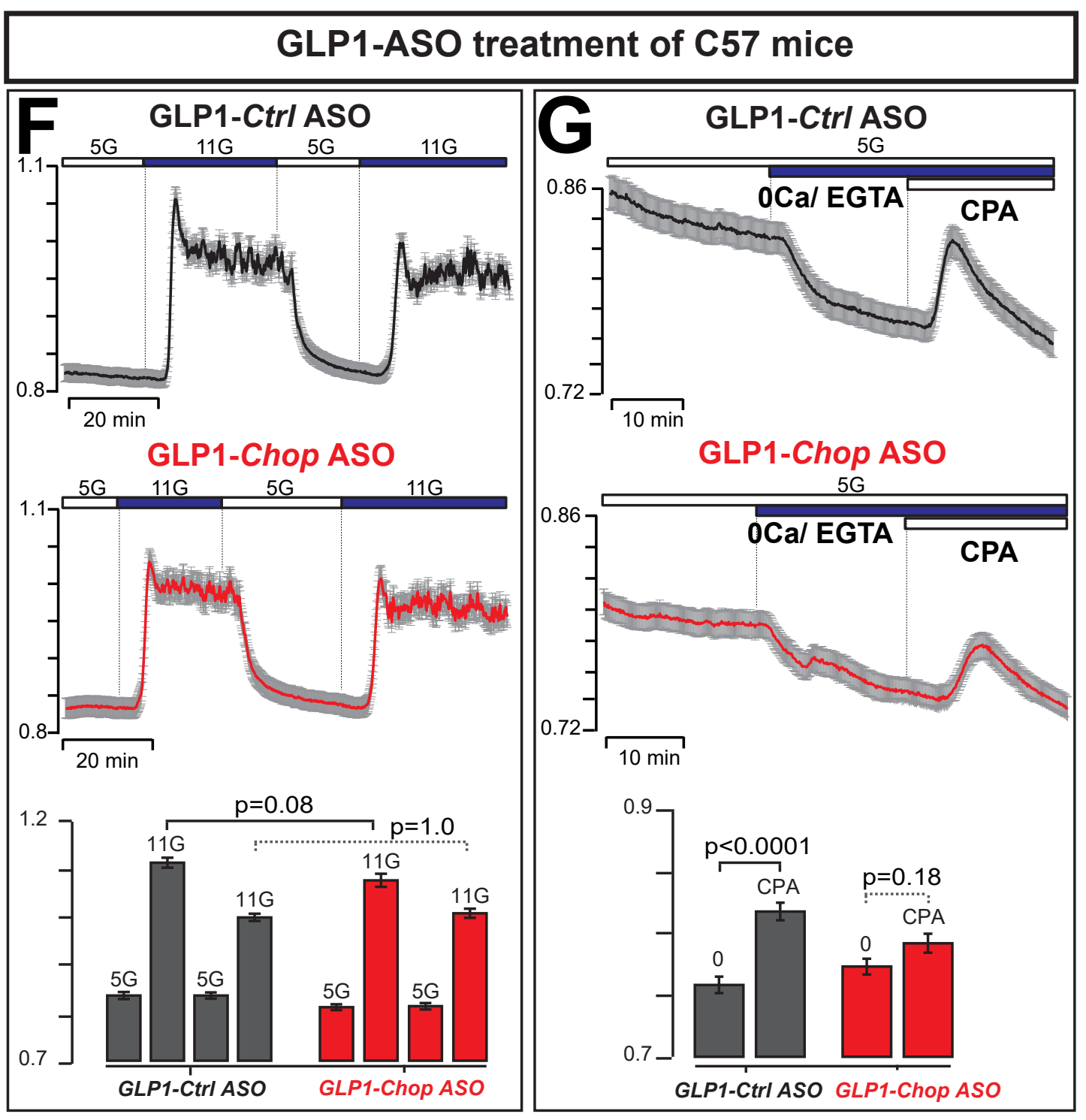

